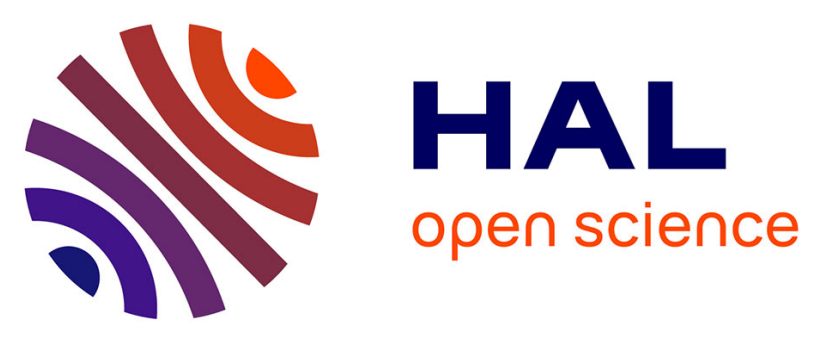

\title{
On the convergence of augmented Lagrangian method for optimal transport between nonnegative densities
}

Romain Hug, Emmanuel Maitre, Nicolas Papadakis

\section{To cite this version:}

Romain Hug, Emmanuel Maitre, Nicolas Papadakis. On the convergence of augmented Lagrangian method for optimal transport between nonnegative densities. Journal of Mathematical Analysis and Applications, 2020, 485 (2), pp.123811. 10.1016/j.jmaa.2019.123811 . hal-01128793v5

\section{HAL Id: hal-01128793 \\ https://hal.science/hal-01128793v5}

Submitted on 13 Sep 2019

HAL is a multi-disciplinary open access archive for the deposit and dissemination of scientific research documents, whether they are published or not. The documents may come from teaching and research institutions in France or abroad, or from public or private research centers.
L'archive ouverte pluridisciplinaire HAL, est destinée au dépôt et à la diffusion de documents scientifiques de niveau recherche, publiés ou non, émanant des établissements d'enseignement et de recherche français ou étrangers, des laboratoires publics ou privés. 


\title{
On the convergence of augmented Lagrangian method for optimal transport between nonnegative densities
}

\author{
Romain $\operatorname{Hug}^{1,4,5}$, Emmanuel Maitre ${ }^{2}$ \& Nicolas Papadakis ${ }^{3}$ \\ ${ }^{1}$ Univ. Grenoble Alpes, CNRS, LJK, 38000 Grenoble, France \\ ${ }^{2}$ Grenoble Institute of Technology, LJK, 38000 Grenoble, France \\ ${ }^{3}$ Univ. Bordeaux, IMB, CNRS, UMR 5251, F-33400 Talence, France. \\ ${ }^{4}$ Johannes Kepler Univ., RICAM, 4040 Linz, Austria. \\ ${ }^{5}$ Univ. Artois, LML, 62307 Lens, France
}

\begin{abstract}
The dynamical formulation of the optimal transport problem, introduced by J. D. Benamou and Y. Brenier [4], corresponds to the time-space search of a density and a momentum minimizing a transport energy between two densities. In order to solve this problem, an algorithm has been proposed to estimate a saddle point of a Lagrangian. We study the convergence of this algorithm in the most general case where initial and final densities vanish on some areas of the transportation domain. Under these conditions, the main difficulty of our study is the proof of existence of a saddle point and of uniqueness of the densitymomentum component, as it leads to deal with non-regular optimal transportation maps. For these reasons, a detailed study of the properties of the velocity field associated to an optimal transportation map in quadratic space is required.
\end{abstract}

\section{Introduction}

The optimal transport problem is generally formulated as follows: considering two sets of particles or probability measures, find the allocation between those discrete or continuous objects while minimizing a given cost. This is referred to as optimal transport or optimal allocation. Even if these two denominations describe the same problem, they reflect two different approaches. Indeed, while it was initially a problem of optimal displacement, the pioneer Gaspard Monge, acknowledging the fact that the optimal trajectory from one point to another is a straight line, reduced this problem to a simple allocation problem. The same holds for the formulation later given by Leonid Kantorovitch: the problem has also been reduced to a single allocation problem of the elements of a given resource to be transported. The trajectories are thus not involved in the transport cost, which only reflects the price to pay to move a mass from one point to another.

The reduction of the optimal transport problem to an allocation problem first makes it easier to tackle theoretically (see [20]). However, when it comes to describe more accurately the optimal allocation map, this formulation is less efficient. Some approaches then choose to reintroduce the notion of displacement: this is the case with the method we deal with in this paper.

The first attempt to link the optimal allocation and optimal displacement problems has been proposed by R. J. McCann [23]. The continuous displacement between two measures $\rho_{0}$ and $\rho_{1}$ has been considered to determine a continuum of intermediate measures, so that the integral sum of local optimal costs (i.e. the distance of Wasserstein step by step) is equal to the global optimal allocation cost between $\rho_{0}$ and $\rho_{1}$. For infinitely close measures, there is indeed no difference between optimal allocation and optimal displacement. 
Relying on this continuous interpolation between densities $\rho_{0}$ and $\rho_{1}$ by densities $\rho_{t}, t \in[0 ; 1]$, Y. Brenier and J.-D. Benamou introduced a continuous formulation of the optimal transport problem [4], originating from fluid mechanics. This amounts to determine directly the evolution of the density $\rho=t \mapsto \rho_{t}$ as well as the velocity field $v$ which advects the density, associated with the optimal map $\nabla \phi$ (see (3)). Such a pair $(\rho, v)$ therefore satisfies a continuity equation.

The optimal map $\nabla \phi$ between $\rho_{0}$ and $\rho_{1}$ can be estimated without relying on the intermediate densities $\rho_{t}$. Efficient second-order algorithms have for instance been proposed [5], using resolutions of the time-continuous Monge-Ampere equation induced by [23]. These direct approaches are nevertheless limited to non-vanishing densities defined on convex supports. We refer to the book of Cuturi and Peyré [25] for an up-to-date contribution to the current state of research on computational optimal transport.

As far as we know, the only numerical method handling the general case is based on the dynamic fluid mechanic method introduced in [4]. The main interest of this dynamic approach is to exploit the physical formulation of the optimal transport problem. Being expressed in terms of fluid mechanics quantities, it makes the model very flexible, and allows generalizations to non balanced problems $[22,9]$ which are relevant for practical applications. The introduction of new physical constraints (anisotropy of the domain, free divergence or rigidity of the velocity field) in the dynamic problem has been a subject of study in [18]. The problem is reformulated as the minimization of a convex, proper, lower semi-continuous (1.s.c) energy and can be solved using convex optimization tools [3, 24]. Before going any further, let us first introduce the dynamic formulation of the optimal transport problem proposed by Benamou and Brenier.

\section{Formulation of the problem and presentation of the algorithm}

\subsection{Monge-Kantorovich problem in $\mathbb{R}^{d}$ for a quadratic cost}

We denote by $|\cdot|$ the Euclidean norm on $\mathbb{R}^{d}$, for all $d \in \mathbb{N}$, and consider two nonnegative densities $\left(\rho_{0}, \rho_{1}\right)$ on $\mathbb{R}^{d}\left(d \in \mathbb{N}^{*}\right)$, with bounded supports and of same mass. The Monge-Kantorovich problem consists in finding an optimal transport plan $T$ between $\rho_{0} \mathcal{L}^{d}$ and $\rho_{1} \mathcal{L}^{d}$ that minimizes

$$
\int_{\mathbb{R}^{d}} d(x, T(x))^{2} \rho_{0}(x) d x
$$

where $d(x, y)$ is a distance on $\Omega$. We write $T \#\left(\rho_{0} \mathcal{L}^{d}\right)$ the push-forward of $\rho_{0} \mathcal{L}^{d}$ by a transport plan $T$. Having $T \#\left(\rho_{0} \mathcal{L}^{d}\right)=\rho_{1} \mathcal{L}^{d}$ means that for any bounded subset $A$ of $\mathbb{R}^{d}, \int_{A} \rho_{0}=\int_{T^{-1}(A)} \rho_{1}$. The quadratic Wasserstein distance $\mathcal{W}_{2}\left(\rho_{0}, \rho_{1}\right)$ is defined by:

$$
\mathcal{W}_{2}\left(\rho_{0}, \rho_{1}\right)^{2}=\inf _{T \#\left(\rho_{0} \mathcal{L}^{d}\right)=\rho_{1} \mathcal{L}^{d}} \int_{\Omega} d(x, T(x))^{2} \rho_{0}(x) d x
$$

In the Euclidean case, where $d(x, y)=|x-y|$, there exists a unique transport map $T$ between $\rho_{0}$ and $\rho_{1}$ that can be written as the gradient of a lower semi-continuous convex potential $\phi$ (Brenier's Theorem [28] p.66) i.e.

$$
\mathcal{W}_{2}\left(\rho_{0}, \rho_{1}\right)^{2}=\int_{\mathbb{R}^{d}}|\nabla \phi(x)-x|^{2} \rho_{0}(x) d x=\inf _{T \#\left(\rho_{0} \mathcal{L}^{d}\right)=\rho_{1} \mathcal{L}^{d}} \int_{\mathbb{R}^{d}}|T(x)-x|^{2} \rho_{0}(x) d x .
$$

In the dynamic formulation introduced by J. -D. Benamou and Y. Brenier in [4], this Monge problem is reformulated as a minimization of a kinetic energy depending on a mass $\rho$ and a velocity field $v$, such that $\rho$ is transported by $v$ from $\rho_{0}$ to $\rho_{1}$. We now detail this new problem. 


\subsection{Convex and augmented lagrangian formulation}

Let $\rho_{0}, \rho_{1} \in L^{2}\left(\mathbb{R}^{d}\right)$ be two probability densities with bounded supports. The dynamic formulation consists in increasing the dimension of the problem by adding a temporal variable $t \in[0,1]$.

Formally, we look for a couple $(\rho, v)$, where $\rho$ denotes a nonnegative density, and $v$ a vector field with values in $\mathbb{R}^{d}$, both defined on $(0,1) \times \Omega$, where $\Omega$ is a bounded open convex set of $\mathbb{R}^{d}$ containing $\operatorname{supp}\left(\rho_{0}\right)$ and $\operatorname{supp}\left(\rho_{1}\right)$. This couple is required to satisfy the continuity equation,

$$
\partial_{t} \rho+\operatorname{div}(\rho v)=0
$$

with no flux boundary conditions for $\rho v$ on $\partial \Omega \times(0,1)$, and with initial and final conditions for $\rho$ on $\Omega$ :

$$
\rho(0, x)=\rho_{0}(x), \rho(1, x)=\rho_{1}(x) .
$$

Among all couples $(\rho, v)$ checking these conditions, we look for a minimizer of the kinetic energy $E(\rho, v)=(1 / 2) \int_{0}^{1} \int_{\Omega}|v|^{2} \rho d x d t$. As $E$ is not convex and the constraint (4) is nonlinear, the authors of [4] proposed as a new variable $m=\rho v$ instead of $v$, and consider the transport cost:

$$
\mathcal{K}(\rho, m)=\int_{0}^{1} \int_{\Omega} \frac{|m(t, x)|^{2}}{2 \rho(t, x)} d x d t
$$

with the corresponding continuity constraint:

$$
\partial_{t} \rho+\operatorname{div}_{x} m=0
$$

that are subject to no flux boundary conditions on $m$ and initial/final conditions (5). The nonnegativity constraint on $\rho$ turns to $\{\rho>0$ or $(\rho, m)=(0,0)\}$ through the change of variable $m=\rho v$. By introducing a Lagrange multiplier $\psi$ to handle the linear constraints (7) together with boundary and initial/final conditions (5), we can write a saddle-point formulation of the problem:

$$
\inf _{(\rho, m)} \sup _{\psi}\left[\int_{(0,1) \times \Omega} \frac{|m|^{2}}{\rho}-\int_{(0,1) \times \Omega}\left(\partial_{t} \psi \rho-\nabla_{x} \psi \cdot m\right)+\int_{\Omega}\left(\psi(0, \cdot) \rho_{0}-\psi(1, \cdot) \rho_{1}\right)\right] .
$$

Another crucial idea in [4] is to encode the non-negativity constraint on $\rho$ by introducing the Legendre transform of $(\rho, m) \mapsto|m|^{2} /(2 \rho)$ :

$$
F(a, b)=\sup _{(\rho, m)}\left(\rho a+\langle m, b\rangle-\frac{|m|^{2}}{2 \rho}\right) \Leftrightarrow F(a, b)= \begin{cases}0 & \text { if } a \leq-|b|^{2} / 2 \\ +\infty & \text { otherwise }\end{cases}
$$

with $(a, b) \in \mathbb{R} \times \mathbb{R}^{d}$. Since the transport cost $\mathcal{K}$ is convex and l.s.c., it is equal to its biconjugate by the Legendre transform. We therefore have $|m|^{2} /(2 \rho)=\sup _{(a, b)}(\rho a+m \cdot b-F(a, b))$. The problem is thus partially linearized with respect to the variables $(\rho, m)$, while the non-linear part $F$ reduces to the indicator function of a convex set. Considering sup-integral and inf-sup inversions, and setting $q=(a, b), \mu=(\rho, m)$ and $Q=(0,1) \times \Omega$, the saddle point problem (8) is reformulated as $\inf _{(\psi, q)} \sup _{\mu} \mathbf{L}(\psi, p, \mu)$ where

$$
\mathbf{L}(\psi, p, \mu)=\chi_{\mathcal{P}}(q)+G(\psi)+\left\langle\mu, \nabla_{t, x} \psi-q\right\rangle_{L^{2}}
$$

with $G(\psi)=\int_{\Omega} \psi(0, \cdot) \rho_{0} d x-\int_{\Omega} \psi(1, \cdot) \rho_{1} d x$ and $\chi_{\mathcal{P}}=0$ if $q \in \mathcal{P}, \chi_{\mathcal{P}}(q)=+\infty$ otherwise, where the paraboloid $\mathcal{P}$ is defined as

$$
\mathcal{P}=\left\{(a, b) \in L^{2}(Q) \times L^{2}(Q)^{d}, a \leq-|b|^{2} / 2\right\} .
$$

The augmented Langrangian is finally given, for some parameter $r>0$, by:

$$
\mathbf{L}_{r}(\psi, q, \mu)=\chi_{\mathcal{P}}(q)+G(\psi)+\left\langle\mu, \nabla_{t, x} \psi-q\right\rangle_{L^{2}}+\frac{r}{2}\left\|\nabla_{t, x} \psi-q\right\|_{L^{2}}^{2} .
$$




\subsection{Benamou-Brenier algorithm}

To solve the saddle point problem associated to (11), the authors of [4] have proposed an algorithm based on a Uzawa method: the ADMM algorithm introduced by M. Fortin and R. Glowinski in [13]. This consists in performing the following steps, starting from $\left(\psi^{n-1}, q^{n-1}, \mu^{n}\right)$ :

Step A: Find the unique $\psi^{n}$ such that $\mathbf{L}_{r}\left(\psi^{n}, q^{n-1}, \mu^{n}\right) \leq \mathbf{L}_{r}\left(\psi, q^{n-1}, \mu^{n}\right), \forall \psi$,

Step B: Find the unique $q^{n}=\left(a^{n}, b^{n}\right)$ such that $\mathbf{L}_{r}\left(\psi^{n}, q^{n}, \mu^{n}\right) \leq \mathbf{L}_{r}\left(\psi^{n}, q, \mu^{n}\right), \forall q$,

Step C: Update $\mu^{n+1}=\left(\rho^{n+1}, m^{n+1}\right)$ as $\mu^{n+1}=\mu^{n}+r\left(\nabla_{t, x} \psi^{n}-q^{n}\right)$.

More precisely, the algorithm breaks down as follows: Step A can be interpreted as a projection on gradient fields. We look for the unique $\psi^{n} \in H^{1}(Q) / \mathbb{R}$ such that:

$$
\forall h \in H^{1}(Q), G(h)+\left\langle\mu^{n}, \nabla_{t, x} h\right\rangle+r\left\langle\nabla_{t, x} \psi^{n}-q^{n-1}, \nabla_{t, x} h\right\rangle=0 .
$$

This corresponds to find $\psi^{n}$ solution of $-r \Delta_{t, x} \psi^{n}=\operatorname{div}_{t, x}\left(\mu^{n}-r q^{n-1}\right)$, with boundary conditions $r \partial_{t} \psi^{n}(0, \cdot)=\rho_{0}-\rho^{n}(0, \cdot)+r a^{n-1}(0, \cdot)$ and $r \partial_{t} \psi^{n}(1, \cdot)=\rho_{1}-\rho^{n}(1, \cdot)+r a^{n-1}(1, \cdot)$, and no flux boundary conditions on $(0,1) \times \partial \Omega$. This is a kind of Helmholtz decomposition. Step B is an $L^{2}$ orthogonal projection on $\mathcal{P}: q^{n}=P_{\mathcal{P}}\left((1 / r) \mu^{n}+\nabla_{t, x} \psi^{n}\right)$. Step C uses the computed gradient of step A to project onto the affine space of constraints (4) and (5).

\subsection{Objectives and related existing works}

The main object of this article is to propose a theoretical framework allowing to answer the three following points: existence of saddle points, uniqueness of saddle points and convergence of the considered algorithm. This study also leads to characterize rigorously some properties of the velocity field associated to an optimal transport plan.

A first study of the Benamou-Brenier algorithm was carried out in [16] for periodic in space boundary condition: $\Omega=\mathbb{T}^{d}$, where $\mathbb{T}^{d}$ denotes the torus in dimension $d$. The strongest assumption in this work concerned the density $\rho^{*}$, solving the problem (6), that had to be larger than a positive constant. This assumption implies in particular a regularity of the associated transport plan. Under such conditions, the potential $\phi$ in (3) is indeed necessarily of class $C^{1}$ with Lipschitz gradient. Caffarelli studied in [7] and [8] the regularity of an optimal transport plan on a convex domain with respect to the regularity of the initial and final densities $\rho_{0}$ and $\rho_{1}$, additionally assumed to be positive. A special case is $\rho_{0}$ and $\rho_{1}$ strictly positive on $\mathbb{T}^{d}$ and belonging to $C^{\alpha, l}\left(\mathbb{T}^{d}\right)$, for some $l \in(0,1)$, and $\alpha \in \mathbb{N}^{d}$. Cordero and Erausquin have shown in [11] that in this case, the optimal transport potential $\phi$ is of class $C^{\alpha, l+2}$ and, for any $t \in[0,1]$, the density $\rho_{t}$ also has a $C^{\alpha, l}$ regularity on $\mathbb{T}^{d}$ and is bounded from below by a strictly positive constant independent of $t$. Under the same assumptions, Guittet showed in [16] the existence of a solution $\left(\rho^{*}, m^{*}\right)$ for the dynamic formulation of optimal transportation; solution from which was proven the existence of a saddle point $\left(\psi^{*}, q^{*}, \mu^{*}\right)$ for the Lagrangian $\mathbf{L}$. However, there was no uniqueness result for the density-momentum couple $\mu^{*}=\left(\rho^{*}, m^{*}\right)$.

A convergence result of the Benamou-Brenier algorithm was also presented in [16]. Nevertheless, this did not explicitly give the strong or weak convergence of $\mu_{n}$, the main component of the triplet $\left(\psi_{n}, q_{n}, \mu_{n}\right)$. Only the strong convergences in $H^{1} / \mathbb{R}$ and $L^{2}$ of the components $\psi_{n}$ and $q_{n}$ were addressed and the proof suffers from an unclear argument related to Cauchy sequences.

More recently, in parallel works to ours, Goldman and Otto [15] also addressed this regularity question for initial and final densities with bounded support that could vanish. The authors nevertheless assume they are smooth and bounded from below by a positive constant on their support, making them generalized indicator functions. 
In this article, we consider a more general framework. The open set $\Omega$ will here be assumed to be convex and bounded, with no flux boundary conditions on the momentum $m$, but more importantly, the density $\rho$ will not be assumed to be bounded from below by a strictly positive constant. We will merely assume that the densities $\rho_{0}$ and $\rho_{1}$ are non-negative elements of $L^{2}(\Omega)$ and potentially without any required smoothness. We will show the existence of a saddle point for the Lagrangian $\mathbf{L}$, solution of the problem (6), as well as the uniqueness among the set of saddle points $\left(\psi^{*}, q^{*}, \mu^{*}\right)$ of the Lagrangian $\mathbf{L}$ of $\mu^{*}=\left(\rho^{*}, m^{*}\right)$, which shows that the density corresponds to the McCann interpolation. The uniqueness result established in this article only concerns the component $\mu$, since, as we will see at the beginning of the section 6 , there is no uniqueness of the saddle points of $\mathbf{L}$ : in fact, the components $\psi$ and $q$ can vary outside the support of $\rho$.

This study also leads us to a new characterization of a velocity field inherent to an optimal transport in $L^{2}$. We give sufficient assumptions on a velocity field $v \in L_{l o c}^{\infty}\left([0,1] \times \mathbb{R}^{d}\right)$ so that a density transported by $v$ is the McCann interpolation of an optimal (unique) transport (Theorem 7.1). These hypotheses are then reduced to the usual characteristics of optimal isotropic transport, in particular straight-line trajectories, constant speed and curl-free.

\subsection{Organization of the paper}

In section 3 we present the different challenges concerning the existence of the saddle points for $\mathbf{L}$, as well as the uniqueness properties. In section 4 , we carry out a preliminary study of the properties of the velocity field. It gives crucial materials for the following three sections, in which we establish the existence of a saddle point (section 5), the uniqueness of the component $\mu=(\rho, m)$ (section 6), and the characterization a minima of a velocity field which represents an optimal transport (section 7 ). In section 8 , we finally show the weak and strong convergence of Benamou-Brenier algorithm towards a saddle point of the Lagrangian $\mathbf{L}$.

\section{Characterization of saddle points and main results}

The main objective of this first part is to directly build a saddle point of $\mathbf{L}$ defined in (11). Let $\rho_{0}$ and $\rho_{1}$ be two probability densities (i.e. non-negative and of integral equal to 1 ) of $L^{2}\left(\mathbb{R}^{d}\right.$ ) with bounded supports, and $\Omega$ be a bounded convex open set of $\mathbb{R}^{d}$. We assume that $\Omega$ is piecewise of class $C^{1}$ and such that $\operatorname{supp}\left(\rho_{0}\right) \cup \operatorname{supp}\left(\rho_{1}\right) \subset \Omega$. In the remaining of this paper, we denote $Q=(0,1) \times \Omega$. For all $r>0$, we write $\mathbf{L}_{r}^{s p}\left(\rho_{0}, \rho_{1}, \Omega\right)$ the set of saddle points of Lagrangian $\mathbf{L}_{r}$ which are elements of $S=H^{1}(Q) / \mathbb{R} \times L^{2}(Q)^{d+1} \times L^{2}(Q)^{d+1}$. In order to characterize saddle points, we now define the set of properties (I) as follows.

Properties (I). A triplet $(\psi, q, \mu) \in S$ satisfies Properties (I) if and only if:

$(\mathrm{I})_{1} \forall q^{\prime} \in \mathcal{P},\left\langle\mu, q^{\prime}-q\right\rangle \leq 0$,

$(\mathrm{I})_{2} \forall h \in H^{1}(Q), \quad G(h)+\left\langle\mu, \nabla_{t, x} h\right\rangle=0$,

(I) $\nabla_{t, x} \psi=q$.

Proposition 3.1. A saddle point $\left(\psi^{*}, q^{*}, \mu^{*}\right) \in S$ of $\mathbf{L}_{r}$ satisfies Properties (I), for all $r \geq 0$.

Sketch of the proof: We first check that for a triplet $\left(\psi^{*}, q^{*}, \mu^{*}\right) \in S$ satisfying the properties (I), we have the relation $\mathbf{L}_{r}\left(\psi, q, \mu^{*}\right) \geq \mathbf{L}_{r}\left(\psi^{*}, q^{*}, \mu^{*}\right) \geq \mathbf{L}_{r}\left(\psi^{*}, q^{*}, \mu\right)$, for all $(\psi, q, \mu) \in S$, which characterizes a saddle point of $\mathbf{L}_{r}$. Conversely, for a saddle point $\left(\psi^{*}, q^{*}, \mu^{*}\right) \in S$ of $\mathbf{L}_{r}$, we can check one by one Properties (I), first by fixing $\psi=\psi^{*}$ and $q=q^{*}$ for (I) $)_{3}$, then $q=q^{*}$ and $\mu=\mu^{*}$ for $(\mathrm{I})_{2}$, and finally $\mu=\mu^{*}$ and $\psi=\psi^{*}$ for $(\mathrm{I})_{1}$. 
Notice that the saddle points of $\mathbf{L}_{r}$ are independent of $r \geq 0$, and therefore the same than $\mathbf{L}=\mathbf{L}_{0}$.

Setting $\mu=(\rho, m)$ and $q=(a, b) \in \mathcal{P}$, see (10), we can reinterpret (I) $)_{1}$ and $(\mathrm{I})_{2}$. Property (I) $)_{1}$ means that if $\mu(t, x)$ is nonzero then it is orthogonal to the paraboloid at point $q(t, x)$, i.e. co-linear to the vector $(1, b(t, x))$. Property $(\mathrm{I})_{1}$ can thus be translated as follows: $\rho \geq 0$, $m=\rho b, \rho\left(a+|b|^{2} / 2\right)=0$. Next, (I) $)_{2}$ corresponds to the mass conservation equation satisfied by $\rho$ and $b$ (i.e. $\partial_{t} \rho+\operatorname{div}_{x}(\rho v)=0$, for $v=b$ ) for the initial and final densities $\rho_{0}$ and $\rho_{1}$.

We now recall that according to Brenier's Theorem ([28] p. 66), there exists a convex potential $\phi$ verifying $\rho_{1} \mathcal{L}^{d}=\nabla \phi \#\left(\rho_{0} \mathcal{L}^{d}\right)$. For the purpose of our study, we need to specify the properties of the potential $\phi$ : we define the property $\left(\Gamma_{1}\right)$ on the potential $\phi$ as follows:

Property $\left(\Gamma_{1}\right)$. $\phi$ and $\phi^{*}$ are convex, continuous and achieve a minimum on $\mathbb{R}^{d}$.

Here $\phi^{*}$ is the Legendre transform of $\phi$. We recall that a convex and continuous function on $\mathbb{R}^{d}$ is locally Lipschitz. We complete Brenier's Theorem as follows.

Proposition 3.2. Let $\rho_{0}$ be a probability density Lebesgue-measurable on $\mathbb{R}^{d}$ and $\nu_{1}$ a probability measure on $\mathbb{R}^{d}$. There exists a potential $\phi: \mathbb{R}^{d} \rightarrow \mathbb{R}$, satisfying the property $\left(\Gamma_{1}\right)$, such that $\nabla \phi \#\left(\rho_{0} \mathcal{L}^{d}\right)=\nu_{1}$.

Sketch of the proof: One can first show that the optimal transport potential $\phi$ given by the Brenier's Theorem (convex, lower semicontinuous and gradient bounded almost everywhere on $\left.\operatorname{supp}\left(\rho_{0} \mathcal{L}^{d}\right)\right)$ is finite and with a bounded gradient on an open neighborhood of the support of $\rho_{0} \mathcal{L}^{d}$. It is then possible to extend the restriction of $\phi$ to this neighborhood by $\bar{\phi}$, a finite convex function on $\mathbb{R}^{d}$ continuous, supralinear and sub-quadratic. The supralinearity of $\bar{\phi}$ implies existence of a global minimum for the latter, and ensures that its Legendre transform $\bar{\phi}^{*}$ is also finite (and thus continuous) on $\mathbb{R}^{d}$. As $\bar{\phi}$ is sub-quadratic, it ensures the supralinearity of $\bar{\phi}^{*}$, and therefore the existence of a global minimum.

Definition 3.1. Let $\rho_{0}$ be a probability density Lebesgue-measurable on $\mathbb{R}^{d}$ and $\nu_{1}$ a probability measure defined on the Lebesgue $\sigma$-algebra in $\mathbb{R}^{d}$. We denote by $\Phi\left(\rho_{0} \mathcal{L}^{d}, \nu_{1}\right)$ the set of functions $\phi: \mathbb{R}^{d} \rightarrow \mathbb{R}$ satisfying the property $\left(\Gamma_{1}\right)$ such that $\nabla \phi \#\left(\rho_{0} \mathcal{L}^{d}\right)=\nu_{1}$.

We can now define from $\phi$ the following quantities.

Definition 3.2. For all $t \in(0,1)$, introducing the potential $\phi_{t}=(1-t) \frac{|\cdot|^{2}}{2}+t \phi$, we define:

1. The characteristic displacement at time $t$,

$$
X_{\phi}(t, \cdot)=(1-t) \mathrm{id}+t \nabla_{x} \phi=\nabla_{x} \phi_{t}
$$

2. The associated velocity field $v$,

$$
v_{\phi}(t, \cdot)=\frac{\mathrm{id}-\nabla_{x}\left(\phi_{t}\right)^{*}}{t}
$$

where $\left(\phi_{t}\right)^{*}$ denotes the Legendre transform of the potential $\phi_{t}$.

3. The density $\rho_{\phi}$, defined as the union for $t \in[0,1]$ of the McCann interpolation densities $\rho_{\phi}^{t}$ between $\rho_{0} \mathcal{L}^{d}$ and $\rho_{1} \mathcal{L}^{d}[23]$ :

$$
\rho_{\phi}(t, \cdot) \mathcal{L}^{d}=\rho_{\phi}^{t} \mathcal{L}^{d}=X_{\phi}(t, \cdot) \#\left(\rho_{0} \mathcal{L}^{d}\right) .
$$

In the following, when there is no ambiguity, we will write $\rho, v$ and $X$ instead of $\rho_{\phi}, v_{\phi}$ and $X_{\phi}$ in order to lighten the notation. 


\subsection{Reformulation of Properties (I)}

In the following, we set $\mu=(\rho, \rho v)$ and $q=\left(-\frac{1}{2}|v|^{2}, v\right)$. To construct a saddle point, $\mu$ and $q$ should satisfy properties (I). It is then sufficient to check that $\rho$ and $v$ defined in (17) and (16) satisfy the following properties:

Definition 3.3. For $n \in \mathbb{N}, 1 \leq p \leq+\infty$, a measurable function $f: Q \longrightarrow \mathbb{R}^{n}$, and a functional space $X$, we write $f \in X$ when each component of $f$ is in $X$. We denote by $W_{l o c}^{1, \infty}([0,1] \times \Omega)$ the space of functions $\psi \in W^{1, \infty}((0,1) \times \omega)$, for all bounded open set $\omega \subset \Omega$.

Properties (II).

(II) 1 The density $\rho$ is an element of $L^{2}(Q)$.

$(\mathrm{II})_{2}$ The velocity field $v$ is an element of $L_{l o c}^{\infty}(\bar{Q})^{d}$.

$(\mathrm{II})_{3}$ The potential $(\rho, v)$ satisfies the mass conservation equation in the distributions sense for the initial and final conditions $\rho_{0}$ and $\rho_{1}$ and no flux conditions:

$$
\forall h \in C^{\infty}(\bar{Q}), \int_{Q}\left(\partial_{t} h+v \cdot \nabla h\right) \rho+\int_{\Omega} h(0, x) \rho_{0}(x) d x-\int_{\Omega} h(1, x) \rho_{1}(x) d x=0
$$

$(\mathrm{II})_{4}$ There exists $\psi \in W_{l o c}^{1, \infty}([0,1] \times \Omega)$ such that $v=\nabla_{x} \psi$.

(II) $)_{5}$ The velocity field $v$ satisfies the Burgers equation in the sense of distributions:

$$
\partial_{t} v+\frac{1}{2} \nabla_{x}|v|^{2}=0
$$

Remark 3.1. These properties are stated in a general case : $\Omega$ is a convex open set of $\mathbb{R}^{d}$ (and $Q=(0,1) \times \Omega)$, which is not necessarily bounded (see Section 7). However, until Section $6, \Omega$ is assumed to be bounded, in context of Benamou-Brenier's Algorithm.

Properties (II $)_{4}$ and (II) $)_{5}$ describe the features of an isotropic optimal transport for a quadratic cost: (II) ${ }_{4}$ corresponds to a curl-free velocity field (without crossing trajectories) and (II) $)_{5}$ is in line with the property of straight-line displacement.

To build a triplet $(\psi, q, \mu)$ satisfying properties (I), it is is sufficient that $\rho$ and $v$ satisfy properties (II). However, having a triplet $(\psi, q, \mu)$ satisfying the properties (I) is not sufficient to build a density-velocity field pair $(\rho, v)$ checking properties (II), and such that $\mu=(\rho, \rho v)$ and $q=\left(-\frac{1}{2}|v|^{2}, v\right)$. Indeed, the component $q$ may not belong to the boundary of the paraboloid $(a, b) \rightarrow a+(1 / 2)|b|^{2} \leq 0$ outside the support of $\mu$.

Properties (II) $)_{1}$ and $(\mathrm{II})_{2}$ ensure that the saddle point is in the correct space $S$. Indeed, we have

$$
\left[q \in L^{\infty}(Q)^{d+1} \Leftrightarrow v \in L^{\infty}(Q)^{d}\right] \text { and }\left[\mu \in L^{2}(Q)^{d+1} \Leftrightarrow \rho \in L^{2}(Q) \text { and } \rho v \in L^{2}(Q)^{d}\right],
$$

and, for the potential $\psi$, we have $W^{1, \infty}(Q) \subset H^{1}(Q)$.

The properties (II) $)_{2}$ and $(\mathrm{II})_{5}$ imply property $(\mathrm{I})_{3}$. Indeed, having $q$ deriving from a spacetime potential amounts to verifying, for a dimension $d \leq 2$, that $\operatorname{curl}_{t, x}(q)=0$ (recalling that $\left.q=\left(-\frac{1}{2}|v|^{2}, v\right)\right)$ in the sense of distributions (see [14] Theorem 2.9 p.31), so that

$$
\left\{\begin{array}{l}
\partial_{t} v+\frac{1}{2} \nabla|v|^{2}=0 \\
\operatorname{curl}_{x}(v)=0 \Leftrightarrow \exists \psi \in \mathcal{D}^{\prime}(Q), v=\nabla_{x} \psi
\end{array}\right.
$$


From Definition of $v$ in (16), we see that the velocity derives from a potential in space in the sense of the distributions, namely:

$$
v(t, \cdot)=\nabla_{x}\left(\frac{1}{t}\left(\frac{1}{2}|\cdot|^{2}-\left(\phi_{t}\right)^{*}\right)\right)
$$

where the potential $\phi$ is an element of $L_{l o c}^{1}(Q)$. This proves property $(\mathrm{I})_{3}$, provided that the field $v$ is an element of $L^{\infty}(Q)^{d}$ and verifies the Burgers equation in the sense of distributions.

Even if the notion of rotational is less easy to cope with in dimension $d>2$, it is always possible to deduce property (I) $)_{3}$ from (II) $)_{2}$ and (II) $)_{5}$ (see [29]), whatever the dimension $d$ is, provided that we have $v \in L^{\infty}(Q)^{d}$. Property (II) 3 corresponds to (I) $)_{2}$. Indeed, we can easily extend by a density argument the relation to $h \in H^{1}(Q)$ once it is established for $h \in C^{\infty}(\bar{Q})$. Finally remark that with the above results, Property $(\mathrm{I})_{1}$ is verified by setting $m=\rho v$.

Remark 3.2. We also notice that, according to (20), (II) $)_{2}$ implies (II) $)_{4}$. Indeed, we can deduce from $(\mathrm{II})_{2}$ that the potential $\psi(t, \cdot)=\frac{1}{t}\left(\frac{1}{2}|\cdot|^{2}-\left(\phi_{t}\right)^{*}\right)$ is in $L_{l o c}^{\infty}(Q)$, and then in $W_{l o c}^{1, \infty}([0,1] \times \Omega)$.

\subsection{Main results of existence, uniqueness and regularity}

We now give our main results of existence and uniqueness on saddle points.

Theorem 3.1 (Existence of a saddle point). Let $\rho_{0}$ and $\rho_{1}$ be two probability densities of $L^{2}\left(\mathbb{R}^{d}\right)$ with bounded supports, and let $\Omega$ be a sufficiently smooth bounded convex open set of $\mathbb{R}^{d}$ such that $\operatorname{supp}\left(\rho_{0}\right) \cup \operatorname{supp}\left(\rho_{1}\right) \subset \Omega$. Then, for all $\phi \in \Phi\left(\rho_{0} \mathcal{L}^{d}, \rho_{1} \mathcal{L}^{d}\right)$ (Definition 3.1), for $\rho=\rho_{\phi}$ and $v=v_{\phi}$ (see (17) and (16) in Definition 3.2), and setting $\mu=(\rho, \rho v)$ and $q=\left(-(1 / 2)|v|^{2}, v\right)$, there exists $\psi \in W_{\text {loc }}^{1, \infty}([0,1] \times \Omega)$ such that $q=\nabla_{t, x} \psi$, and $(\psi, q, \mu)$ is a saddle point of Lagrangian $\mathbf{L}$ (or at least its restriction on $(0,1) \times \Omega$ ).

The density $\rho$ is in $C^{0}\left([0,1], L^{2}(\Omega)\right)$, and the velocity field $v$ is locally Lipschitz on the space $(0,1) \times \mathbb{R}^{d}$, and satisfies properties $(\mathrm{II})_{4}$ and $(\mathrm{II})_{5}$. Moreover, $v \in W^{1, p}((0,1) \times \Omega)$ for all $1 \leq p<2$, and $\nabla_{t, x} v \in L^{\infty}\left(0,1 ; L^{1}(\Omega)\right)$.

Having $\nabla_{t, x} v \in L^{\infty}\left(0,1 ; L^{1}(\Omega)\right)$ is a result in itself, and it is not directly used to characterize an optimal transport velocity field in $L^{2}$. Very close properties are nevertheless considered to show the statements on the uniqueness of the component $(\rho, m)$ of the saddle points of $\mathbf{L}$.

Theorem 3.2 (Uniqueness of density and momentum). If the triplet $\left(\psi^{*}, q^{*}, \mu^{*}\right)$ is a saddle point of $\mathbf{L}$ (the assumptions on $\rho_{0}, \rho_{1}$ and $\Omega$ being the same as in Theorem 3.1), then, for any potential $\phi \in \Phi\left(\rho_{0} \mathcal{L}^{d}, \rho_{1} \mathcal{L}^{d}\right)$, with $\rho=\rho_{\phi}$ and $v=v_{\phi}$, we have $\mu^{*}=\left(\rho^{*}, m^{*}\right)=(\rho, \rho v)$, and

$$
\rho(t, \cdot) \mathcal{L}^{d}=X(t, \cdot) \#\left(\rho_{0} \mathcal{L}^{d}\right) \in C\left([0,1], L^{2}(\Omega)\right), \text { with } X(t, \cdot)=\nabla_{x} \phi_{t}=(1-t) \text { id }+t \nabla_{x} \phi .
$$

In general, the set of saddle points $(\psi, q, \mu)$ of $\mathbf{L}$ is not reduced to a single element: only the component $\mu=(\rho, m)$ is unique. In other words, the set of points $(\psi, q, \mu)$ of $\mathbf{L}$ shares the same component $\mu$ and there is uniqueness of the density $\rho$ and the velocity field $v$ on the support of $\rho$. The components $q$ and $\psi$ can vary outside the support of $\rho$. To prove these two results, we now study the properties of a velocity field $v$ defined as in (16), for $\phi$ satisfying property $\left(\Gamma_{1}\right)$.

\section{$4 \quad$ First velocity field properties}

In this section, we present properties on velocity fields associated to optimal transport maps and namely show $(\mathrm{II})_{2}$ and $(\mathrm{II})_{5}$. These results constitute the basis of existence and uniqueness results concerning the saddle points of $\mathbf{L}$, as well as the generalized results of section 7 . 
We recall that for a function $f: \mathbb{R}^{n} \rightarrow \mathbb{R}$ proper, l.s.c. and convex, the proximal operator $\operatorname{Prox}_{f}(x)$ of $f$ at $x \in \mathbb{R}^{n}$ is the unique minimizer of $f+\frac{1}{2}|x-\cdot|^{2}$, so that $y=\operatorname{Prox}_{f}(x) \Leftrightarrow$ $x-y \in \partial f(y)$. The operators $\operatorname{Prox}_{f}$ and id $-\operatorname{Prox}_{f}$ are non-expansive (1-Lipschitz), and the proximal operator of $f^{*}$, the Legendre transform of $f$, satisfies Moreau's identity $\operatorname{Prox}_{\gamma f^{*}}=$ id $-\gamma \operatorname{Prox}_{f / \gamma}(\cdot / \gamma)$. The $\gamma$-Moreau envelope ${ }^{\gamma} f$ is defined as the inf-convolution of $f$ by $1 /(2 \gamma)|\cdot|^{2}$.

\subsection{Definition and first properties of the velocity field}

From (16), the velocity field of an optimal transport can be written as a proximal operator $\mathbf{p}$. This makes easier to deal with the problems of "breaks" of the velocity field (which are not necessarily discontinuities).

Let $\phi: \mathbb{R}^{d} \mapsto \mathbb{R}$ satisfy property $\left(\Gamma_{1}\right)$, i.e. $\phi$ is convex and continuous at every point of $\mathbb{R}^{d}$, and admits in each of these points a non-empty and compact sub-differential. According to the properties linking Legendre conjugation and inf-convolution, if $t \in(0,1)$, by setting $\phi_{t}=(1-t)|\cdot|^{2} / 2+t \phi$, then the Legendre conjugation $\left(\phi_{t}\right)^{*}$ is of class $C^{1}$ on $\mathbb{R}^{d}$, and for all $(t, x) \in[0,1) \times \mathbb{R}^{d}$ we define the operator $\mathbf{p}$ by

$$
\mathbf{p}(t, x)=\operatorname{Prox}_{\frac{t}{1-t} \phi}\left(\frac{x}{1-t}\right)=\frac{\mathrm{id}-\operatorname{Prox}_{(1-t)(t \phi)^{*}}}{1-t}=\nabla_{x}\left(\phi_{t}\right)^{*}
$$

The velocity $v=v_{\phi}$ introduced in (16) can be defined from $\mathbf{p}$ by $v(t, x)=[x-\mathbf{p}(t, x)] / t$, for all $t \in(0,1)$ and $x \in \mathbb{R}^{d}$. When there is no ambiguity on $\phi$, we use $v$ to denote the velocity field $v$. For all $t \in[0,1), \mathbf{p}(t, \cdot)$ is $1 /(1-t)$-Lipschitz (non-expansiveness of operator Prox), and

$$
\forall x, y \in \mathbb{R}^{d}, y=\mathbf{p}(t, x)=\nabla_{x}\left(\phi_{t}\right)^{*}(x) \Leftrightarrow x \in \partial_{x}\left(\phi_{t}\right)(y)=(1-t) y+t \partial \phi(y) .
$$

From these definitions and using $X$ as introduced in (15), we get:

$$
y=\mathbf{p}(t,(1-t) y+t \nabla \phi(y))=\mathbf{p}(t, X(t, y)) \text { i.e. } \nabla \phi(y)-y=\partial_{t} X(t, y)=v(t, X(t, y)) .
$$

Given that $X(t, \cdot)=\nabla \phi_{t}$ and $\mathbf{p}(t, \cdot)=\nabla_{x}\left(\phi_{t}\right)^{*}$, then for any $t \in(0,1)$, and for all $x \in \mathbb{R}^{d}$ such that $\phi$ Frechet-differentiable in $x$, we have $\left(\nabla_{x}\left(\phi_{t}\right)^{*} \circ \nabla \phi_{t}\right)(x)=x$. Consequently, we observe that $\mathbf{p}(t, \cdot)$ formally represents the inverse of the characteristic trace $X(t, \cdot)=\nabla_{x}\left(\phi_{t}\right)$. It would really be the case if $\phi$ was of class $C^{1}$ with a Lipschitz gradient. In the general case (i.e. $\phi$ is not $C^{1}$ and only checks property $\left.\left(\Gamma_{1}\right)\right), \mathbf{p}(t, \cdot)$ is nevertheless not injective on $\mathbb{R}^{d}$. The operator $\mathbf{p}(t, \cdot)$ thus repairs the "breaks" that can be generated by a transport plan. Indeed, $\mathbf{p}(t, \cdot)$ re-concentrates the areas generated by diffusion (by the characteristic trajectories $X(t, \cdot)$ ) of the break points on these same points.

Next, we deduce that for any fixed $t \in(0,1)$, the velocity field $v(t, \cdot)$ is Lipschitz on $\mathbb{R}^{d}$. It is also possible to define a Lipschitz constant that is only time dependent and does not depend on $\phi$.

Lemma 4.1. For all $\in(0,1), L_{t}=2 /(t(1-t))$ is a Lipschitz constant for $v(t, \cdot)$ on $\mathbb{R}^{d}$ (for the Euclidean norm $|\cdot|)$, whatever $\phi$ is.

With Rademacher's Theorem, the velocity field $v$ is therefore continuous and Fréchet-differentiable almost everywhere in space, and thus $\left\|\nabla_{x} v(t, x)\right\|$ is additionally uniformly bounded by $L_{t}=2 /(t(1-t))$ for almost all $x \in \mathbb{R}^{d}$, where $\|\cdot\|$ denotes the subordinate norm to $|\cdot|$.

Remark 4.1. The "break" points of the transport plan correspond to the points where the potential $\phi$ is not differentiable. Although Rademacher's Theorem ensures that the set of such points is negligible, the diffusion of these breaks, and in particular the torsions/high variations of the 
velocity field at these points in $t=0$ (or the re-connections at $t=1$ for the points of irregularity of $\left.\phi^{*}\right)$ is not. Indeed, the torsions of the velocity field in the neighborhood of break points may induce a loss of $H^{1}$ regularity of the velocity field. Having a $H^{1}$ regularity on the potential $\phi$ would greatly simplify the study on the uniqueness of the saddle points of the Lagrangian $\mathbf{L}$ presented in section 6. Unfortunately, such regularity can not be obtained in general.

\subsection{Velocity field control}

Lemma 4.2 (Property $\left.(\mathrm{II})_{2}\right)$. For $\phi$ satisfying property $\left(\Gamma_{1}\right)$, the velocity field $v=v_{\phi}$ (defined from $\phi$ as in (16)) satisfies $v \in L_{l o c}^{\infty}\left([0,1] \times \mathbb{R}^{d}\right)^{d}$.

Sketch of the proof: We can show that $v(t, \cdot)$ is uniformly bounded on any bounded open set $\omega \subset \mathbb{R}^{d}$ in the neighborhood of $t=0$. Take for instance $t \in(0,1 / 2]$ and $y \in \omega$, and let $x \in(1-t) y+t \partial \phi(y)$. According to $(22)$, we have $\mathbf{p}(t, x)=y$, so $v(t, x)=(x-y) / t \in \partial \phi(y)-y$. Because of Lemma 4.1, for all $t \in(0,1)$, we have:

$$
|v(t, y)-v(t, x)| \leq|v(t, x)|+\frac{2}{t(1-t)}|x-y| \leq 4\left|\frac{x-y}{t}\right|=4|v(t, x)| .
$$

We have $|v(t, y)| \leq 5|v(t, x)| \leq 5\left(\sup _{x \in \omega}|\partial \phi(x)|+\sup (\omega)\right)<+\infty$. Indeed, with the property $\left(\Gamma_{1}\right), \phi$ and $\phi^{*}$ are assumed to be finite and convex on $\mathbb{R}^{d}$ and therefore locally Lipschitz. The same argument can be used in the neighborhood of $t=1$ (using $\phi^{*}$ in place of $\phi$ ).

Moreover, since $v(t, \cdot)=\left(\mathrm{id}-\nabla_{x}\left(\phi_{t}\right)^{*}\right) / t$, we can prove (see for instance [29]) that there exists $\psi \in W_{l o c}^{1, \infty}([0,1] \times \Omega)$ (which is not necessarily equal to $|\cdot|^{2} / 2-\phi_{t}^{*}$ ), such that $v=\nabla_{x} \psi$.

As already stated, for every $t \in(0,1), v(t, \cdot)$ is continuous and Lipschitz on $\mathbb{R}^{d}$ with constant $2 / t(1-t)$. The field $v(t, \cdot)$ is therefore Lipschitz on $\mathbb{R}^{d}$, for a Lipschitz constant independent of $t$ on any interval $[\alpha, \beta] \subset(0,1)$. One can for instance consider the constant $M_{\alpha, \beta}=\sup _{[\alpha, \beta]} 2 / t(1-t)$. It is therefore possible to apply the Cauchy-Lipschitz Theorem on $[\alpha, \beta]$.

Then, for every $x \in \mathbb{R}^{d}$ and $t \in(0,1)$, the Cauchy problem: $y_{t, x}^{\prime}=v\left(\cdot, y_{t, x}\right)$, with $y_{t, x}(t)=x$, admits a unique maximum solution over any interval $(\alpha, \beta), 0<\alpha<t<\beta<1$. We can then prove that there exists a unique solution defined on $(0,1)$ and that it can be written $y_{t, x}(s)=(s-t) v(t, x)+x$ for all $s \in(0,1)$. Indeed, such a solution satisfies $y_{t, x}(t)=x$, and $y_{t, x}^{\prime}(s)=v(t, x)=v(s,(s-t) v(t, x)+x)=v\left(s, y_{t, x}(s)\right)$. By using the properties (22) of the operator $\mathbf{p}$, we can show that, for all $t, s \in(0,1)$, and for all $x \in \mathbb{R}^{d}$, we have

$$
v(t, x)=v(s,(s-t) v(t, x)+x)
$$

This relation implies that $v$ is a time-space locally Lipchitz function: we can bring back any difference between two space-times values of $v$ to a same time, and apply the space Lipchitz property of $v$ for fixed time. This property is also shown for example in [28].

Proposition 4.1 (Property (II) $\left.)_{5}\right)$. With the property $\left(\Gamma_{1}\right)$, $v$ satisfies the Burgers' equation (19), that is to say, in the distribution sense:

$$
\partial_{t} v+\frac{1}{2} \nabla_{x}|v|^{2}=0
$$

which is a generalized form of $\partial_{t} v+v \cdot \nabla_{x} v=0$.

The proof is given Appendix A [19]. In the case where $\phi$ is sufficiently regular (case where there is no "breaks" in the transport), then $\nabla_{x} \phi_{t}$ is invertible for $t \in(0,1)$, and we directly find that $0=\partial_{t t} \nabla_{x} \phi_{t}=\left(\partial_{t} v+v \cdot \nabla_{x} v\right)\left(\nabla_{x} \phi_{t}\right)$, since $\nabla_{x} \phi-\mathrm{id}=\partial_{t} \nabla_{x} \phi_{t}=v\left(t, \nabla_{x} \phi_{t}\right)$. 


\section{$5 \quad$ Existence of a saddle point (Proof of Theorem 3.1)}

In order to prove the existence of a saddle point for the Lagrangian $\mathbf{L}$, we have built a couple density-velocity field $(\rho, v)$ satisfying conditions (II). The velocity field $v=v_{\phi}$, immediately checks properties (II) $)_{2}$ (Lemma 4.2) and (II) $)_{5}$ (subsection 4.2). From (20) and (II) $)_{2}, v$ verifies (II) $)_{4}$ (see Remark 3.2).

We now have to build a density $\rho=\rho_{\phi}$, verifying property (II) $)_{1}$, such that the pair $(\rho, v)$ satisfies the mass conservation of $(\mathrm{II})_{3}$. The candidate density is naturally the one formed by the set of intermediate measurements between $\rho_{0} \mathcal{L}^{d}$ and $\rho_{1} \mathcal{L}^{d}=\left(\nabla \phi \# \rho_{0} \mathcal{L}^{d}\right)$, which can be assimilated to a series of "optimal micro-transports" along the time scale $[0,1]$ and correspond to the interpolation of McCann (17), defined at each time $t$ by the density $\rho_{t}=\rho_{t}^{\phi}$ of the measure

$$
\rho_{t} \mathcal{L}^{d}=\rho_{t}^{\phi} \mathcal{L}^{d}=[(1-t) \mathrm{id}+t \nabla \phi] \#\left(\rho_{0} \mathcal{L}^{d}\right)=\nabla \phi_{t} \#\left(\rho_{0} \mathcal{L}^{d}\right) .
$$

For all $1<p<+\infty$, McCann also defines in [23] the "internal energy" of the space $\left(\mathcal{P}_{2}\left(\mathbb{R}^{d}\right), \mathcal{W}_{p}\right)$ as

$$
\mathcal{F}_{p}(\mu)=\left\{\begin{array}{l}
\int_{\mathbb{R}^{d}}|f(x)|^{p} d \mathcal{L}^{d}(x) \text { if } \mu=f \cdot \mathcal{L}^{d} \in \mathcal{P}_{2}\left(\mathbb{R}^{d}\right), \\
+\infty \text { otherwise }
\end{array}\right.
$$

where $\mathcal{P}_{2}\left(\mathbb{R}^{d}\right)$ is defined as the space of probability measures $\mu$ on $\mathbb{R}^{d}$ satisfying the condition $\int_{\mathbb{R}^{d}}|x|^{2} d \mu(x)<+\infty$ (any finite measure on $\mathbb{R}^{d}$ with compact support is an element of $\mathcal{P}_{2}\left(\mathbb{R}^{d}\right)$ ). This energies are convex along the geodesics of the space $\left(\mathcal{P}_{2}\left(\mathbb{R}^{d}\right), \mathcal{W}_{p}\right)$, which are the interpolations of McCann. Thus, the function $\Lambda_{p}:[0,1] \rightarrow[0,+\infty]$, defined for all $t \in[0,1]$ as $\Lambda_{p}(t)=\mathcal{F}_{p}\left(\rho_{t} \mathcal{L}^{d}\right)$, for $t \mapsto \rho_{t}$ defined in $(26)$, is convex on [0,1], and is moreover finite in $t=0$ and $t=1$, since $\rho_{0}, \rho_{1} \in L^{p}\left(\mathbb{R}^{d}\right)$, and $\rho_{0} \mathcal{L}^{d}, \rho_{1} \mathcal{L}^{d} \in \mathcal{P}_{2}\left(\mathbb{R}^{d}\right)$. Hence it is finite and bounded on the whole interval $[0,1]$. Therefore, by definition of $\Lambda_{p}$, we have $\rho_{t} \in L^{p}\left(\mathbb{R}^{d}\right)$ for every $t \in[0,1][1]$. Moreover, $t \mapsto\left\|\rho_{t}\right\|_{L^{p}\left(\mathbb{R}^{d}\right)}$ is bounded on $[0,1]$ by a constant $M$. We observe that $t \mapsto \rho_{t}$ is weakly continuous in $L^{p}\left(\mathbb{R}^{d}\right)$ on $[0,1]$. Indeed, $t \mapsto \rho_{t} d \mathcal{L}^{d}$ is continuous from $[0,1]$ to $\mathcal{D}^{\prime}\left(\mathbb{R}^{d}\right)$ : it is then sufficient to use the density of $C_{c}^{0}\left(\mathbb{R}^{d}\right)$ in $L^{q}\left(\mathbb{R}^{d}\right)$, for $q \in(1,+\infty)$ such that $1 / p+1 / q=1$. Since the function $\Lambda_{p}$ is convex and finite on $[0,1], t \mapsto \Lambda(t)=\left\|\rho_{t}\right\|_{L^{p}\left(\mathbb{R}^{d}\right)}^{p}$ is continuous on $(0,1)$ and admits a right limit at $t=0$ and a left limit at $t=1$. Thus, the application $t \mapsto \rho_{t}$ is strongly continuous from $[0,1]$ to $L^{p}\left(\mathbb{R}^{d}\right)$ [29]. Conversely, one can characterize the McCann interpolation by the relation

$$
\forall h \in C_{c}^{0}\left([0,1] \times \mathbb{R}^{d}\right), \int_{(0,1) \times \mathbb{R}^{d}} h \rho d x \otimes d t=\int_{0}^{1} \int_{\mathbb{R}^{d}} h(t, t \nabla \phi(x)+(1-t) x) \rho_{0}(x) d x d t .
$$

Indeed, using Fubini's Theorem, it can be shown that for any density $\rho$ satisfying (28), there exists a family of densities $\left(\rho_{t}\right)_{t \in[0,1)}$ of McCann's interpolations measures between $\rho_{0} \mathcal{L}^{d}$ and $\rho_{1} \mathcal{L}^{d}$, such that $t \mapsto \rho_{t} \in C^{0}\left([0,1), L^{p}\left(\mathbb{R}^{d}\right)\right)$ and $(t, x) \mapsto \rho_{t}(x)$ be measurable, and such that $\rho(t, x)=\rho_{t}(x)$ for almost all $(t, x) \in[0,1) \times \mathbb{R}^{d}[17]$.

From Brenier's Theorem ([28] p. 66), we have $\operatorname{supp}\left(\rho_{1}\right)=\overline{\nabla \phi\left(\operatorname{supp}\left(\rho_{0}\right)\right)}$. This property also holds for potentials satisfying the property $\left(\Gamma_{1}\right)$ considered in Proposition 3.2. Thus, for $\Omega$ a convex open set of $\mathbb{R}^{d}$ containing $\operatorname{supp}\left(\rho_{0}\right)$ and $\operatorname{supp}\left(\rho_{1}\right)$, we have the inclusion $(t \nabla \phi+(1-$ $t)$ id $)\left(\operatorname{supp}\left(\rho_{0}\right)\right) \subset \Omega$, for all $t \in[0,1]$.

Therefore, the candidate density $\rho:(t, x) \mapsto \rho_{t}(x)$, defined in (26), satisfies the condition (II) ${ }_{1}$. The component $\mu=(\rho, \rho v)$ is zero in the neighborhood of the space boundary, and thus verifies the Neumann conditions implicitly included in the weak form of mass conservation (II $)_{3}$. 
Finally, the definition immediately implies that for all $h \in C^{\infty}(\bar{Q})$,

$$
\begin{aligned}
& \int_{0}^{1} \int_{\mathbb{R}^{d}}\left(\partial_{t} h+v \cdot \nabla_{x} h\right) \rho d x d t=\int_{0}^{1} \int_{\mathbb{R}^{d}}\left(\partial_{t} h(t, X(t, \cdot))+\partial_{t} X(t, \cdot) \cdot \nabla_{x} h(t, X(t, \cdot))\right) \rho_{0} d x d t \\
= & \int_{0}^{1} \frac{d}{d t} \int_{\mathbb{R}^{d}} h(t, X(t, \cdot)) \rho_{0} d x d t=\int_{\mathbb{R}^{d}} h\left(1, \nabla_{x} \phi(x)\right) \rho_{0}(x) d x-\int_{\mathbb{R}^{d}} h(0, x) \rho_{0}(x) d x .
\end{aligned}
$$

Since $\rho_{1} \mathcal{L}^{d}=\nabla \phi \#\left(\rho_{0} \mathcal{L}^{d}\right)$, we have $\int_{\mathbb{R}^{d}} h\left(1, \nabla_{x} \phi\right) \rho_{0} d x=\int_{\mathbb{R}^{d}} h(1, \cdot) \rho_{1} d x$. The integrals are well defined as $v \in L^{\infty}(Q)$. Then, the pair $(\rho, v)$ satisfies the condition (II) $)_{3}$. Moreover, by taking $q \leq 2$ such that $1 / p+1 / q=1$, the weak relation of mass consevation extends to the test functions $h \in W^{1, q}(Q)$. We can now prove the Theorem.

Proof of Theorem 3.1: Let us recall that an element $(\psi, q, \mu)$ of $\mathbf{L}^{s p}\left(\rho_{0}, \rho_{1}, \Omega\right)$ must satisfy $(\psi, q, \mu) \in$ $S$, as well as the properties (I) and (II).

$(\mathrm{I})_{1}$ From $(\mathrm{II})_{1}$ and $(\mathrm{II})_{2}$, we know that $v \in L^{\infty}(Q)^{d}$ and $t \mapsto \rho(t, \cdot)=X(t, \cdot) \# \rho_{0} \in C^{0}\left([0,1], L^{2}(\Omega)\right)$. Then $\mu=(\rho, \rho v), q=\left(-(1 / 2)|v|^{2}, v\right) \in L^{2}(Q)$, and $\operatorname{supp}(\mu) \subset \operatorname{supp}(\rho) \subset[0,1] \times \Omega$. Spatial homogeneous Neumann conditions of $\mu$ are thus verified.

Moreover, by setting $\mu=(\rho, \rho v)$ and $q=\left(-(1 / 2)|v|^{2}, v\right)$, the condition (I) 1 is naturally verified as for all $q^{\prime}=(a, b) \in \mathcal{P}=\left\{(a, b), a \leq-|b|^{2} / 2\right\}$, the paraboloid defined in (10), we have

$$
\begin{aligned}
\left\langle\mu, q^{\prime}-q\right\rangle & =\int_{0}^{1} \int_{\Omega}(a \rho+b \cdot v \rho) d x d t-\int_{0}^{1} \int_{\Omega} \frac{1}{2}|v|^{2} \rho d x d t \\
& \leq \int_{0}^{1} \int_{\Omega}\left(a+\frac{1}{2}|b|^{2}+\frac{1}{2}|v|^{2}\right) \rho d x d t-\int_{0}^{1} \int_{\Omega} \frac{1}{2}|v|^{2} \rho d x d t \leq 0 .
\end{aligned}
$$

$(\mathrm{I})_{2}$ The condition $(\mathrm{I})_{2}$ results from the conservation of mass $(\mathrm{II})_{3}$ : satisfied by the pair $(\rho, v)$.

$(\mathrm{I})_{3}$ According to Proposition 4.1, $v$ satisfies the Burgers equation (II) $)_{5}$ in the sense of distributions. Moreover, from $(\mathrm{II})_{4}$, we know that $v$ derives from a spatial potential. It gives us the existence of one $\psi \in W^{1, \infty}(Q)$ such that $q=\nabla_{t, x} \psi$. Property $(\mathrm{I})_{3}$ is thus checked.

We finally obtain that the triplet $(\psi, q, \mu)$ is an element of

$$
W^{1, \infty}(Q) / \mathbb{R} \times L^{\infty}(Q)^{d+1} \times L^{2}(Q)^{d+1} \subset S .
$$

From the property $\psi \in W^{1, \infty}(Q)$, the field $v=\nabla_{x} \psi$ then satisfies the properties (II) $)_{4}$ and (II) $)_{5}$. The final regularity properties of $v_{\phi}$ given in Theorem 3.1 come from three other results proven in Appendix [19]): the velocity field $v$ is locally Lipschitz on the space $(0,1) \times \mathbb{R}^{d}$, the property $\nabla_{t, x} v \in L^{\infty}\left(0,1 ; L^{1}(\Omega)\right)$, and its corollary $v \in W^{1, p}((0,1) \times \Omega)$ for all $1 \leq p<2$.

\section{$6 \quad$ Uniqueness properties of saddle points}

The uniqueness of the density $\rho$ has been proven with the energetic formulation, via the uniqueness of the geodesics in Wasserstein's space $\mathcal{W}_{2}$ (see [27] Proposition 5.32). We nevertheless here propose a proof based on the structure of the velocity field and its regularity properties. More precisely, we use a DiPerna-Lions method [12], in which the insufficient regularity of the velocity field $\left(W^{1, p}, p<2\right.$, rather than $\left.H^{1}\right)$ is compensated by the specific structure of the velocity field of an optimal transport. The regularity of the velocity field provides (a minima) the regularity of the solution of the dual transport equation of the mass conservation equation. 


\subsection{Uniqueness of the velocity field on the density support}

We first study the uniqueness of the velocity field on the support of the different candidate densities. We show that for any saddle points of $\mathbf{L}$, denoted by $\left(\psi^{*}, q^{*}, \mu^{*}\right)=\left(\psi^{*}, q^{*},\left(\rho^{*}, m^{*}\right)\right)$, the densities $\rho^{*}$ are transported with the same velocity field $v$.

Lemma 6.1. We consider $\Omega$ a bounded convex open set of $\mathbb{R}^{d}$, and $\rho_{0}, \rho_{1} \in L^{2}\left(\mathbb{R}^{d}\right)$ two densities which supports are included in $\Omega$. If the triplet $\left(\psi^{*}, q^{*}, \mu^{*}\right)$ is an element of $\mathbf{L}^{s p}\left(\rho_{0}, \rho_{1}, \Omega\right)$ with $\mu^{*}=\left(\rho^{*}, m^{*}\right)$, then, for all $\phi \in \Phi\left(\rho_{0} \mathcal{L}^{d}, \rho_{1} \mathcal{L}^{d}\right)$, we have $m^{*}=\rho^{*} v$, with $v=v_{\phi}$ defined in (16).

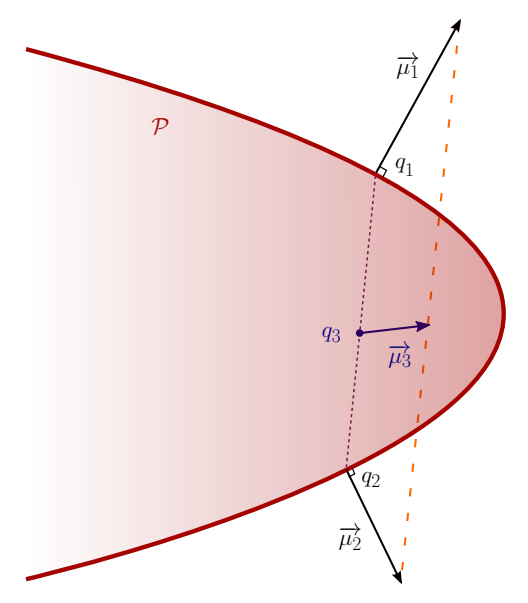

Figure 1: Illustration of the characterization of the saddle points of $\mathbf{L}$.

Sketch of the proof: Following Figure 1, we give a "schematic" proof of the uniqueness of the velocity field on the union of supports of the candidate densities, which is based on the convexity of the set of saddle points and the strict convexity of the paraboloid $\mathcal{P}$ defined in (10). For a more rigorous proof we refer to [17] (chapter 4).

We recall that $Q=(0,1) \times \Omega$. We assume $\left(\psi_{1}, q_{1}, \mu_{1}\right)$ and $\left(\psi_{2}, q_{2}, \mu_{2}\right)$ to be two saddle points of $\mathbf{L}$. The fields $\mu_{1}$ and $\mu_{2}$ are both orthogonal in the sense of the canonical scalar product of $L^{2}$ to the paraboloid $\mathcal{P}$, respectively at points $q_{1}$ and $q_{2}$, which imply a pointwise orthogonality almost everywhere. We will see in section 8 , that the set of saddle points of $\mathbf{L}$ is convex so that the $\lambda\left[\left(\psi_{1}, q_{1}, \mu_{1}\right)+(1-\lambda)\left(\psi_{2}, q_{2}, \mu_{2}\right)\right]$ is also a saddle point of $\mathbf{L}$, for all $\lambda \in[0,1]$. Then, for almost all $(t, x) \in Q$ and for all $\lambda \in[0,1]$, the vector $\left[\lambda \mu_{1}+(1-\lambda) \mu_{2}\right](t, x)$ is also orthogonal to $\mathcal{P}$ at point $\left[\lambda q_{1}+(1-\lambda) q_{2}\right](t, x)$ which is strictly inside $\mathcal{P}$ if $q_{1}(t, x) \neq q_{2}(t, x)$. Then we have $\left[\lambda \mu_{1}+(1-\lambda) \mu_{2}\right](t, x)=0$ for all $\lambda \in[0,1]$, i.e. $\mu_{1}(t, x)=\mu_{2}(t, x)=0$. Therefore, almost everywhere, when $\left(\mu_{1}(t, x), \mu_{2}(t, x)\right) \neq(0,0)$, we have $q_{1}(t, x)=q_{2}(t, x)$, and then $\mu_{1}(t, x)$ and $\mu_{2}(t, x)$ are colinear. We can conclude if one of these two saddle points is the one we have constructed above.

To sum up: for any fixed $\phi \in \Phi\left(\rho_{0} \mathcal{L}^{d}, \rho_{1} \mathcal{L}^{d}\right)$ and for every saddle point $\left(\psi^{*}, q^{*}, \mu^{*}\right)$ of $\mathbf{L}, \rho^{*}$ is associated with the same velocity field $v$ with $\partial_{t} \rho^{*}+\operatorname{div}_{x}\left(\rho^{*} v\right)=0$ with the initial and final conditions $\rho^{*}(0, \cdot)=\rho_{0}$ and $\rho^{*}(1, \cdot)=\rho_{1}$. In other words, for all $h \in H^{1}(Q)$, we have

$$
\int_{0}^{1} \int_{\Omega}\left(\partial_{t} h(t, x)+v(t, x) \cdot \nabla_{x} h(t, x)\right) \rho^{*}(t, x) d x d t+\int_{\Omega} h(0, \cdot) \rho_{0}-\int_{\Omega} h(1, \cdot) \rho_{1}=0 .
$$

We now prove that there exists a unique $\rho^{*}$ which satisfies these conditions, that is to say $\rho^{*}(t, \cdot)=X(t, \cdot) \# \rho_{0}$, with $X=X_{\phi}$ as defined in (15), for all $\phi \in \Phi\left(\rho_{0} \mathcal{L}^{d}, \rho_{1} \mathcal{L}^{d}\right)$. 


\subsection{Uniqueness of density in $L^{2}$}

As the velocity field $v$ is unique on the union of the supports of the candidate densities, the uniqueness of the density $\rho$ implies the uniqueness of the momentum $m=\rho v$. The next proposition is the main ingredient to show the uniqueness of $\rho$.

Proposition 6.1. Let $\phi: \mathbb{R}^{d} \rightarrow \mathbb{R}$ a convex potential satisfying property $\left(\Gamma_{1}\right), \rho_{0} \in L^{2}\left(\mathbb{R}^{d}\right)$ with a bounded support, and consider a velocity field $v=v_{\phi}$ defined from $\phi$ as $(16)$. If $\rho \in L^{2}\left((0,1) \times \mathbb{R}^{d}\right)$ is a density with bounded support in $[0,1] \times \mathbb{R}^{d}$, such that

$$
\left\{\begin{array}{l}
\partial_{t} \rho+\operatorname{div}_{x}(\rho v)=0 \\
\rho(0, \cdot)=\rho_{0}
\end{array}\right.
$$

in the sense of distributions, i.e.

$$
\forall h \in C_{c}^{\infty}\left([0,1) \times \mathbb{R}^{d}\right), \int_{0}^{1} \int_{\mathbb{R}^{d}}\left(\partial_{t} h+v \cdot \nabla_{x} h\right) \rho d x d t+\int_{\mathbb{R}^{d}} h(0, \cdot) \rho_{0} d x=0,
$$

then $\rho(t, \cdot)=\rho_{\phi}(t, \cdot)=\left(t \nabla \phi+(1-t)\right.$ id) $\# \rho_{0}$ for almost all $t \in[0,1]$ (as defined in (17)). In other words:

$$
\forall \varphi \in C_{c}^{0}\left((0,1) \times \mathbb{R}^{d}\right), \int_{0}^{1} \int_{\mathbb{R}^{d}} \varphi \rho d x d t=\int_{0}^{1} \int_{\mathbb{R}^{d}} \varphi(t, t \nabla \phi(x)+(1-t) x) \rho_{0}(x) d x d t .
$$

Moreover $t \mapsto \rho(t, \cdot) \in C^{0}\left([0,1), L^{2}\left(\mathbb{R}^{d}\right)\right)$.

Proof: Let $\Omega$ be a bounded open set of $\mathbb{R}^{d}$ such that $\operatorname{supp}\left(\rho_{0}\right)$ is included in $\Omega$ and $\operatorname{supp}(\rho) \subset$ $[0,1] \times \Omega$, and let $Q=(0,1) \times \Omega$. Let $(\psi, q, \mu)$ be a saddle point of $\mathbf{L}$ as defined in (11), and let $\phi \in \Phi\left(\rho_{0} \mathcal{L}^{d}, \rho_{1} \mathcal{L}^{d}\right)$ (thus checking Property $\left(\Gamma_{1}\right)$ on $\left.\mathbb{R}^{d}\right)$. The triplet $(\psi, q, \mu)$ satisfies the properties (I), which implies in particular the weak mass conservation $G(h)+\left\langle\mu, \nabla_{t, x} h\right\rangle=0$ for all $h \in H^{1}(Q)$, as well as the linear relation between momentum and density: $\mu=(\rho, m)=$ $(\rho, \rho v) \in L^{2}(Q)$, with $v=v_{\phi}$ defined in (16) and satisfying the properties (II) $)_{4}$ and (II) $)_{5}$ (see subsection 3.2). From these properties, we deduce that for every $h \in H^{1}(Q)$ :

$$
\int_{0}^{1} \int_{\Omega}\left(\partial_{t} h+v \cdot \nabla_{x} h\right) \rho d x d t=\int_{\Omega} h(1, \cdot) \rho_{1} d x-\int_{\Omega} h(0, \cdot) \rho_{0} d x
$$

Let $\varphi \in C_{c}^{\infty}\left((0,1) \times \mathbb{R}^{d}\right)$ such that $\operatorname{supp}(\varphi) \subset(0,1) \times \Omega \subset Q$. By solving the transport problem in $v$ and $\varphi$ with a characteristics method, we consider the function $\mathfrak{h}$ defined for any $(t, x) \in(0,1) \times \mathbb{R}^{d}$ by:

$$
\mathfrak{h}(t, x)=-\int_{t}^{1} \varphi(s,(s-t) v(t, x)+x) d s
$$

which satisfies

$$
\partial_{t} \mathfrak{h}+v \cdot \nabla_{x} \mathfrak{h}=\varphi \quad \text { and } \quad \nabla_{t, x} \mathfrak{h}={ }^{t}\left(\nabla_{t, x} v\right) \alpha+\beta,
$$

with $\alpha \in L^{\infty}(Q)^{d}$, and $\beta \in L_{l o c}^{\infty}(Q)^{d+1}$. We also have $\mathfrak{h}(1, \cdot)=0$ and $\mathfrak{h}(0, \cdot)=-\int_{0}^{1} \varphi(t, X(t, \cdot)) d t$ with $X(t, \cdot)=t \nabla \phi+(1-t)$ id.

To solve the problem, it would be sufficient to take $\mathfrak{h}$ in (32) as a test function. Unfortunately, the velocity field only satisfies $\nabla_{t, x} v \in L^{\infty}\left(0,1 ; L^{1}(\Omega)\right)$ and $v \in W^{1, p}((0,1) \times \Omega)$ for all $1 \leq p<2$ (see Appendix A [19]), thus $v=v_{\phi} \in \mathcal{H}:=\cap_{1 \leq p<2} W^{1, p}(Q)$. The function $\mathfrak{h}$ is thus an element of $\mathcal{H}$. Since we do not have a better integrability than $L^{2}$ on $\rho$, we cannot extend the space of test functions of $(32)$ to a larger space than $H^{1}(Q)$ as $\mathcal{H}$. The counter-example of Caffarelli on the strict division of the mass show that, in general, the field $v$ is not an element of $H^{1}(Q)$. 
We then choose to approximate $\mathfrak{h}$ by regularizing the velocity field $v$ associated to the transport plan $\nabla \phi$ with $v_{\gamma}=v_{\gamma_{\phi}}$ associated to the regularized transport plans $\nabla^{\gamma} \phi$, where ${ }^{\gamma} \phi$ denotes the $\gamma$-regularization of the potential $\phi$ by a Moreau's envelope (see beginning of section 4). This regularization has the property of erasing the breaks of the transport plan, which are responsible for the fact that $v$ does not have regularity $H^{1}$ in the neighborhood of $t=0$.

With a characteristics method, it is thus possible to construct some test functions $\mathfrak{h}_{\gamma} \in$ $H^{1}(Q)$, uniformly zero in the neighborhood of $t=1$ (independently of $\gamma$ ), such that:

$$
\partial_{t} \mathfrak{h}_{\gamma}+v_{\gamma} \cdot \nabla_{x} \mathfrak{h}_{\gamma}=\varphi=\partial_{t} \mathfrak{h}_{\gamma}+v \cdot \nabla_{x} \mathfrak{h}_{\gamma}+\left(v_{\gamma}-v\right) \cdot \nabla_{x} \mathfrak{h}_{\gamma}
$$

and such that $\mathfrak{h}_{\gamma}(0, \cdot)$ converges to $-\int_{0}^{1} \varphi\left[t,(1-t)\right.$ id $\left.+t \nabla_{x} \phi\right] d t$ in $L^{2}\left(\mathbb{R}^{d}\right)$ when $\gamma$ tends to 0 . By injecting such a function $\mathfrak{h}_{\gamma}$ in (32), one obtains

$$
\begin{gathered}
\int_{0}^{1} \int_{\mathbb{R}^{d}} \varphi \rho d x d t=\int_{0}^{1} \int_{\mathbb{R}^{d}} \varphi\left[t,(1-t) x+t \nabla_{x} \phi(x)\right] \rho_{0}(x) d x d t+R_{\gamma}(\varphi), \text { with } \\
R_{\gamma}(\varphi)=\int_{0}^{1} \int_{\mathbb{R}^{d}}\left(v_{\gamma}-v\right) \cdot \nabla_{x} \mathfrak{h}_{\gamma} \rho d x d t-\int_{\mathbb{R}^{d}} \mathfrak{h}_{\gamma}(0, \cdot) \rho_{0} d x-\int_{0}^{1} \int_{\mathbb{R}^{d}} \varphi\left[t,(1-t) \mathrm{id}+t \nabla_{x} \phi\right] \rho_{0} d x d t .
\end{gathered}
$$

In order to prove Proposition 6.1 , it is therefore necessary to show that $R_{\gamma}(\varphi)$ converges to 0 when $\gamma$ tends to 0 . This makes use of the results of the Appendix A [19].

The $\mathfrak{h}_{\gamma}$ are defined with respect to $v_{\gamma}$ by (33). We can then prove from (34) that we have,

$$
\left|\nabla_{x} \mathfrak{h}_{\gamma}(t, x)\right| \leq\left(\left|\nabla_{x} v_{\gamma}(t, x)\right|+1\right)\left\|\nabla_{x} \varphi\right\|_{L^{\infty}\left([0,1] \times \mathbb{R}^{d}\right)}, \text { for almost all }(t, x) \in(0,1) \times \mathbb{R}^{d} .
$$

For more details, we refer to [17] (subsection 4.2.5), in which it is proven that $\mathfrak{h}_{\gamma} \in H^{1}(Q)$.

The velocity field $v_{\gamma}$ can be extended by continuity in $t=0$. It is the same for $\mathfrak{h}_{\gamma}$, which is continuous on $[0,1) \times \bar{\Omega}$, and for all $x \in \mathbb{R}^{d}$, one has:

$$
\mathfrak{h}_{\gamma}(0, x)=-\int_{0}^{1} \varphi\left(s, s v_{\gamma}(0, x)+x\right) d s=-\int_{0}^{1} \varphi\left(s, s \nabla^{\gamma} \phi(x)+(1-s) x\right) d s
$$

which coincides with the trace $L^{2}$ of $\mathfrak{h}_{\gamma}$ in $t=0$. We can show that $\nabla^{\gamma} \phi(x)$ converges for almost all $x \in \Omega$ to $\nabla \phi(x)$ (see Lemma 4 in the Appendix A [19]), for all $x$ where $\phi$ is differentiable.

In addition, for all $(s, x) \in(0,1) \times \Omega$, the term $\varphi\left(s, s \nabla^{\gamma} \phi(x)+(1-s) x\right)$ is uniformly bounded by $\|\varphi\|_{L^{\infty}}$. Thus, by dominated convergence, we have

$$
r_{\gamma}(\varphi)=\int_{\Omega} \mathfrak{h}_{\gamma}(0, \cdot) \rho_{0} d x+\int_{0}^{1} \int_{\Omega} \varphi(s, s \nabla \phi(x)+(1-s) x) \rho_{0}(x) d x d s \underset{\gamma \rightarrow 0}{\longrightarrow} 0 .
$$

Let $t_{m} \in(0,1)$ such that $\operatorname{supp}(\varphi) \subset\left(0, t_{m}\right) \times \Omega$. From (37), we thus have

$$
\left|R_{\gamma}(\varphi)\right| \leq\left|r_{\gamma}(\varphi)\right|+\left\|\nabla_{x} \varphi\right\|_{L^{\infty}}\left(\int_{0}^{t_{m}} \int_{\Omega}\left|v-v_{\gamma}\right| \cdot\left|\nabla_{x} v_{\gamma}\right| \cdot|\rho| d x d t+\int_{0}^{t_{m}} \int_{\Omega}\left|v-v_{\gamma}\right| \cdot|\rho| d x d t\right) .
$$

We can prove that $\left|v-v_{\gamma}\right|$ is uniformly bounded and simply converges to 0 on $(0,1) \times \Omega$ when $\gamma$ tends to 0 . Thus, since $\rho \in L^{2}((0,1) \times \Omega)$, we conclude via dominated convergence that the term $\int_{0}^{t_{m}} \int_{\Omega}\left|v-v_{\gamma}\right| \cdot|\rho| d x d t$ converges to 0 .

Finally, to complete the proof of Proposition 6.1, we have to show that $\int_{0}^{t_{m}} \int_{\Omega}\left|v-v_{\gamma}\right| \cdot\left|\nabla_{x} v_{\gamma}\right| \cdot$ $|\rho| d x d t$ converges to 0 . This is the subject of the following Lemma 6.2, which proof will end the one of the current Proposition 6.1. 
Lemma 6.2. Let $\phi: \mathbb{R}^{d} \rightarrow \mathbb{R}$ be a convex potential verifying the property $\left(\Gamma_{1}\right)$. We consider a velocity field $v=v_{\phi}$ defined as in (16) and $0<t_{m}<1$. Let $\rho \in L^{2}\left((0,1) \times \mathbb{R}^{d}\right)$, with bounded support into $[0,1] \times \mathbb{R}^{d}$. Let $\Omega$ a bounded open set of $\mathbb{R}^{d}$ such that $\operatorname{supp}\left(\rho_{0}\right) \subset \Omega$ and $\operatorname{supp}(\rho) \subset[0,1] \times \Omega$. For any $\gamma>0$, we define $v_{\gamma}=v_{\gamma_{\phi}}$, where ${ }^{\gamma} \phi$ is the Moreau envelope of $\phi$ by the parameter $\gamma$ (see beginning of section 4). Then we have the result of convergence:

$$
\int_{0}^{t_{m}} \int_{\Omega}\left|v-v_{\gamma}\right| \cdot\left|\nabla_{x} v_{\gamma}\right| \cdot|\rho| d x d t \underset{\gamma \rightarrow 0}{\longrightarrow} 0 .
$$

Before giving a sketch of proof of the previous Lemma, let us state two results concerning the control of the regularized velocity fields $v_{\gamma}$. The first one is an important uniform regularity result for the velocity field and its regularization.

Proposition 6.2. We assume that $\phi$ satisfies the property $\left(\Gamma_{1}\right)$. Let $R^{\prime}>R>0$ and $a \in \mathbb{R}^{d}$ such that $\phi(a)=\inf _{\mathbb{R}^{d}} \phi$ and let $M=\sup _{x \in B(a, 2(R+|a|))}|\partial \phi(x)|$.

Then there exists a constant $C>0$, independent of $\phi, \gamma, a, R$ and $R^{\prime}$, such that for all $t_{0} \in(0,1)$ satisfying the condition $t_{0}<\min \left\{1 / 2,\left(R^{\prime}-R\right) /(M+2|a|)\right\}$, and by setting $v_{0}=v$, we have the property:

$$
\forall \gamma \geq 0, \forall t \in\left(0, t_{0}\right], \int_{B(a, R)}\left|\nabla_{x} v_{\gamma}(t, x)\right| d x \leq \frac{C}{t_{0}\left(1-t_{0}\right)} \mathcal{L}^{d}\left(B\left(a, R^{\prime}\right)\right) .
$$

Thus $\nabla_{x} v_{\gamma} \in L^{\infty}\left(0, t_{0} ; L^{1}(B(a, R))\right)$, for all $\gamma \geq 0$.

The proof of this proposition is the main object of Appendix A [19].

Remark 6.1. Proposition 6.2 is an important result on the control of the velocity field of a dynamical optimal transport: in particular, we have $\nabla_{t, x} v \in L^{p}\left(0,1 ; L_{l o c}^{q}\left(\mathbb{R}^{d}\right)\right)$ for all $1<p \leq$ $+\infty$ and $1 \leq q<+\infty$ such that $1 / p+1 / q>1$, especially $\nabla_{t, x} v \in L^{\infty}\left(0,1 ; L^{1}(\Omega)\right)$ and $v \in$ $W_{\text {loc }}^{1, p}\left([0,1] \times \mathbb{R}^{d}\right)$ for all $1 \leq p<2$.

Corollary 6.1. Let $\phi$ satisfy the property $\left(\Gamma_{1}\right)$ and $\Omega \subset \mathbb{R}^{d}$ be a bounded open set and $0<t_{m}<1$. Then there exists a constant $K>0$ such that for every $\gamma>0$ and any $t \in\left[0, t_{m}\right]$, we have

$$
\int_{\Omega}\left|\nabla_{x} v_{\gamma}(t, x)\right| d x \leq K
$$

Proof: For $t<t_{0}$, we apply Proposition 6.2; and for $t_{m} \geq t>t_{0}$ we use the fact that the term $\left|\nabla_{x} v\left(t_{0}, \cdot\right)\right|_{1}$ is bounded by $c / t(1-t)$, for $c$ a constant depending only on the chosen norm.

Sketch of proof of Lemma 6.2: For any convex, 1.s.c. proper funtion $f$, the operator id $-\operatorname{Prox}_{\gamma f}$ is non-expansive and $\nabla\left({ }^{\gamma} f\right)(x) \in \partial f\left(\operatorname{Prox}_{\gamma f}(x)\right)$. Moreover, there exists a constant $C>0$ such that for all $t \in(0,1)$,

$$
\left\|\nabla_{x} v(t, \cdot)\right\|_{L^{\infty}\left(\mathbb{R}^{d}\right)} \leq \frac{C}{1-t}\left(\left\|\mathrm{D}^{2} \phi\right\|_{L^{\infty}\left(\mathbb{R}^{d}\right)}+d\right) .
$$

Hence there exists a constant $c$, independent of $\gamma$, such that for every $1 \geq \gamma>0$ and $t \in(0,1)$

$$
\left\|\nabla_{x} v_{\gamma}(t, \cdot)\right\|_{L^{\infty}\left(\mathbb{R}^{d}\right)} \leq \frac{c}{1-t} \min \left\{\frac{1}{t}, \frac{1}{\gamma}\right\} .
$$

By partially bounding $\left|\nabla_{x} v_{\gamma}\right|_{1}$ from above (i.e. we bound only $a^{\beta}$ when $a=a^{\alpha} a^{\beta}, 1=\alpha+\beta$ ), and applying Corollary 6.1, we can show that the convergence resut (40) is true for all $p>2$ such that $\rho \in L^{p}(Q)$, and that there exists a constant $M$ such that:

$$
\forall \gamma \in(0,1], \int_{0}^{t_{m}} \int_{\Omega}\left|v-v_{\gamma}\right| \cdot\left|\nabla_{x} v_{\gamma}\right| \cdot|\rho| d x d t \leq M\|\rho\|_{L^{2}(Q)} .
$$

Therefore, by density of $L^{p}(Q)$ in $L^{2}(Q),(40)$ is also true for $p=2$. 


\subsection{Proof of Theorem 3.2}

To finish the proof of Theorem 3.2, we only have to show that a density $\rho^{*}$ associated to one of the saddle points $\left(\psi^{*}, q^{*}, \mu^{*}\right)$ of $\mathbf{L}$ verifies the conditions of Proposition 6.1.

Proof. Let $\left(\psi^{*}, q^{*}, \mu^{*}\right)$ be a saddle point of $\mathbf{L}$. According to Proposition 3.1, we have $G(h)+$ $\left\langle\mu^{*}, \nabla_{t, x} h\right\rangle=0$, for all $h \in H^{1}(Q)$. Let $\phi \in \Phi\left(\rho_{0} \mathcal{L}^{d}, \rho_{1} \mathcal{L}^{d}\right)$, thus verifying property $\left(\Gamma_{1}\right)$. Following Lemma 6.1 , by defining $v=v_{\phi}$ on $(0,1) \times \mathbb{R}^{d}$ as in (16), we have $m^{*}=\rho^{*} v$. Hence for all $h \in H^{1}(Q)$ :

$$
\int_{Q}\left(\partial_{t} h+v \cdot \nabla_{x} h\right) \rho^{*} d x d t+\int_{\Omega} h(0, \cdot) \rho_{0} d x-\int_{\Omega} h(1, \cdot) \rho_{1} d x=0 .
$$

Let $\overline{\rho^{*}} \in L^{2}\left((0,1) \times \mathbb{R}^{d}\right)$ be the extension in 0 of $\rho^{*}$ on $(0,1) \times \mathbb{R}^{d}$. Notice that for any test function $h \in H_{l o c}^{1}\left((0,1) \times \mathbb{R}^{d}\right)$ we have $h_{\mid Q} \in H^{1}(Q)$ and $\nabla_{t, x} h_{\mid Q}=\left(\nabla_{t, x} h\right)_{\mid Q}$. Hence the relation (45) can be extended from $Q$ to the entire space $(0,1) \times \mathbb{R}^{d}$. According to Proposition 6.1 , we thus have the equivalence $\overline{\rho^{*}}(t, \cdot)=\rho_{\phi}(t, \cdot)=(t \nabla \phi+(1-t)$ id $) \# \rho_{0}$ for almost all $t \in[0,1]$, with in addition $t \mapsto \rho_{\phi}(t, \cdot) \in C^{0}\left([0,1), L^{2}\left(\mathbb{R}^{d}\right)\right)$.

\section{Characterization of an optimal transport velocity field}

We now present a generalization of our study on the uniqueness of the component densitymomentum $\mu$ that characterizes less formally an optimal transport velocity field. The result means that the velocity field has to satisfy properties (II) $)_{4}$ and (II) $)_{5}$ and can be understood as follows.

Any density of $L^{2}$, with bounded support, and advected by a locally bounded divergence free velocity field, whose trajectories are all straight lines (and in particular never intersect), corresponds to an optimal transport, i.e. an interpolation of McCann, and is the only solution for such a displacement.

For a convex open set $\Omega$ of $\mathbb{R}^{d}$, we define the space ${ }^{b} L_{+}^{2}((0,1) \times \Omega)$ of densities $\rho \in L^{2}((0,1) \times \Omega)$ which are non-negative and with compact supports into $[0,1] \times \Omega$.

Theorem 7.1. Let $Q=(0,1) \times \Omega, \Omega$ being a convex open set of $\mathbb{R}^{d}$ not necessarily bounded. Let $v^{*}$ be a velocity field on $\Omega$ satisfying properties (II $)_{4}$ and $(\mathrm{II})_{5}$ and $\rho_{0} \in L^{2}(\Omega)$, with $\rho_{0} \geq 0$ and $\operatorname{supp}\left(\rho_{0}\right)$ bounded in $\Omega$. Let $\rho^{*} \in{ }^{b} L_{+}^{2}(Q)$ be a solution in the sense of distributions of

$$
\left\{\begin{array}{l}
\partial_{t} \rho+\operatorname{div}_{x}\left(\rho v^{*}\right)=0 \\
\rho(0, \cdot)=\rho_{0}
\end{array}\right.
$$

Then the density $\rho^{*}$ is the unique solution of problem (46) in the space ${ }^{b} L_{+}^{2}(Q)$ and we have $\rho^{*} \in C^{0}\left([0,1), L^{2}(\Omega)\right)$. Moreover, there exists a unique non-negative Radon measure $\nu_{1}$ on $\Omega$, which support is bounded in $\bigcup_{t \in[0,1]} \operatorname{supp}\left(\rho^{*}(t, \cdot)\right)$ and a convex function $\phi$ on $\mathbb{R}^{d}$ verifying property $\left(\Gamma_{1}\right)$, such that $\nu_{1}=\nabla \phi \#\left(\rho_{0} \mathcal{L}^{d}\right)$ and

$$
\forall t \in[0,1),\left(\rho^{*}(t, \cdot) \mathcal{L}^{d}\right)=\left(\rho(t, \cdot) \mathcal{L}^{d}\right)=(t \nabla \phi+(1-t) \mathrm{id}) \#\left(\rho_{0} \mathcal{L}^{d}\right),
$$

(with $\rho=\rho_{\phi}$ defined in (17)) which makes the link with McCann's interpolation. The couple $\left(\rho^{*}, v^{*}\right)$ is then solution of

$$
\left\{\begin{array}{l}
\partial_{t} \rho^{*}+\operatorname{div}_{x}\left(\rho^{*} v^{*}\right)=0, \\
\rho^{*}(0, \cdot)=\rho_{0}, \rho^{*}(1, \cdot)=\nu_{1}
\end{array}\right.
$$


which gives in the weak sense

$$
\forall h \in C_{c}^{\infty}([0,1] \times \Omega), \int_{0}^{1} \int_{\Omega}\left(\partial_{t} h+v^{*} \cdot \nabla_{x} h\right) \rho^{*} d x d t+\int_{\Omega} h(0, \cdot) \rho_{0} d x-\int_{\Omega} h(1, \cdot) d \nu_{1}=0 .
$$

Finally, for all $(t, x) \in \operatorname{supp}\left(\rho^{*}\right)$, we have $v^{*}(t, x)=v_{\phi}(t, x)$, defined in (16). The field $v^{*}$ thus satisfies the properties of the velocity field $v$ on $\operatorname{supp}\left(\rho^{*}\right)$. In particular, $v$ is locally Lipschitz on $(0,1) \times \mathbb{R}^{d}$ and it satisfies $\nabla_{t, x} v \in L^{\infty}\left(0,1 ; L_{\text {loc }}^{1}\left(\mathbb{R}^{d}\right)\right)$ and $v \in W_{\text {loc }}^{1, p}\left([0,1] \times \mathbb{R}^{d}\right)$ for all $1 \leq p<2$.

Sketch of the proof: The proof gathers elements from sections 5 and 6 . It is technical, as the final measure (46) is no longer a density measure, of type $\rho_{1} \mathcal{L}^{d}$, but simply a finite Radon measure $\nu_{1}$. We only give the main steps of the proof and refer to [17] (section 4.3) for details.

The first step consists in proving the existence, in the sense of distributions, of the final measure $\nu_{1}$, as defined in the statement of the Theorem. For this purpose we use classical functional analysis tools such as the Riesz Representation Theorem [26]. We also show that the weak formulation (49) is always valid for test functions taken from $W_{c}^{1, \infty}([0,1] \times \Omega)$.

Next we consider $\phi$, an optimal transport potential between $\rho_{0} \mathcal{L}^{p}$, and $\nu_{1}$ satisfying property $\left(\Gamma_{1}\right)$. We take a saddle point $(\mu, q, \psi)$, as done in section 5 and built in Theorem 3.1. As Brenier's Theorem only assumes density for the initial density $\rho_{0} \mathcal{L}^{d}$, Proposition 3.2 is still valid. This is also the case for all inductions done while building the velocity field $v=v_{\phi}$. However, we can not obtain $\rho=\rho_{\phi} \in L^{2}(Q)$, since it requires $\nu_{1}=\rho_{1} \mathcal{L}^{d}$ with $\rho_{1} \in L^{2}(\Omega)$. Hence, we can not extend the test functions of the weak formulation of the mass conservation for the pair $(\rho, v)$ to the space $H_{l o c}^{1}(Q)$, which only considers absolutely continuous initial measures. Extending test functions to the space $W_{l o c}^{1, \infty}(Q)$ is required as the potential $\psi$ necessarily belongs to this space.

We then build a second saddle point from the pair $\left(\rho^{*}, v^{*}\right)$, i.e. a triplet $\left(\mu^{*}, q^{*}, \psi^{*}\right)$, with $\mu^{*}=\left(\rho^{*}, \rho^{*} v^{*}\right), q^{*}=\left(-(1 / 2)\left|v^{*}\right|^{2}, v^{*}\right)$ and $\nabla_{t, x} \psi^{*}=q^{*}$. We can then, as well as for Lemma 6.1, prove the uniqueness of the velocity field on the supports of $\rho$ and $\rho^{*}$, i.e. $\rho^{*} v^{*}=\rho^{*} v$. Although our triplets $(\mu, q, \psi)$ and $\left(\mu^{*}, q^{*}, \psi^{*}\right)$ are not necessarily in $L^{2}$, and we can no longer speak of "projections" and "orthogonality" as in the schematic proof of Lemma 6.1, the reasoning remains globally the same and we reach the same conclusion (Lemma 4.3-14 of [17]). Thus, according to Proposition 6.1, the density $\rho^{*}$ verifies the relation $(47)$, with $t \mapsto \rho^{*}(t, \cdot) \in C^{0}\left([0,1), L^{2}(\Omega)\right)$.

Now, let us show that $\rho^{*}$ is the only solution with bounded support of the problem (46) in the space ${ }^{b} L_{+}^{2}(Q)$. Assume there exists two solutions $\rho^{1}, \rho^{2}$ of $(46)$ in ${ }^{b} L_{+}^{2}(Q)$. Then the density $\bar{\rho}=\left(\rho^{1}+\rho^{2}\right) / 2$ is still a solution in ${ }^{b} L_{+}^{2}(Q)$. Therefore, there would exist a convex function $\bar{\phi}$ of $\mathbb{R}^{d}$ satisfying property $\left(\Gamma_{1}\right)$, such that, by defining $v_{\bar{\phi}}$ as in (16), we have $\bar{\rho} v^{*}=\bar{\rho} v_{\bar{\phi}}$, i.e. $\left(\rho^{1}+\rho^{2}\right) v^{*}=\left(\rho^{1}+\rho^{2}\right) v_{\bar{\phi}}$. The field $v^{*}$ is then almost everywhere equal to the field $v_{\bar{\phi}}$ on $\operatorname{supp}\left(\rho^{1}\right) \cup \operatorname{supp}\left(\rho^{2}\right)$, thus $\rho^{1} v^{*}=\rho^{1} v_{\bar{\phi}}$ and $\rho^{2} v^{*}=\rho^{2} v_{\bar{\phi}}$. Therefore, $\rho^{1}$ and $\rho^{2}$ both solves problem (46), by replacing $v^{*}$ with $v_{\bar{\phi}}$. According to Proposition 6.1 , we thus have $\rho^{1}=\rho^{2}$ : $t \mapsto \rho_{\bar{\phi}}=(t \nabla \bar{\phi}+(1-t)$ id $) \# \rho_{0}$ in $\left.L^{2}\left((0,1) \times \mathbb{R}^{d}\right)\right)$, and then in $L^{2}(Q)$.

\section{Convergence of the algorithm}

The convergence of Benamou-Brenier algorithm to a saddle point of the Lagrangian $\mathbf{L}$ is shown.

Proposition 8.1. $(\psi, q, \mu)$ is a saddle point of the Lagrangian $\mathbf{L}$ defined in (9) if and only if it is a fixed point of the Benamou-Brenier algorithm (12)-(14).

Proof: Let $(\psi, q, \mu)$ be a saddle point of $\mathbf{L}$ defined in (9). We denote by $\left(\psi^{\prime}, q^{\prime}, \mu^{\prime}\right)$ the new triplet obtained after one iteration of the algorithm. Let us show that $\left(\psi^{\prime}, q^{\prime}, \mu^{\prime}\right)=(\psi, q, \mu)$ in $S$. From property $(\mathrm{I})_{2}$ of (I), and taking $h=\psi^{\prime}-\psi$, we obtain $\left\|\nabla_{t, x}\left(\psi^{\prime}-\psi\right)\right\|^{2}=0$ in step A (12). 
According to the Poincaré inequality, we get $\psi^{\prime}=\psi$ in $H^{1}((0,1) \times \Omega) / \mathbb{R}$. In step B (13), we look for the unique $q^{\prime}$ verifying $\left\langle\mu+\nabla \psi^{\prime}-q^{\prime}, p-q^{\prime}\right\rangle \leq 0$, for all $p \in \mathcal{P}$. From Properties (I) 1 and (I) 3 which characterize a saddle point, we get $\psi=\psi^{\prime}$. Having $q^{\prime}=q$ is a good candidate for $q^{\prime}$ and therefore the only one. Finally, $\nabla_{t, x} \psi^{\prime}=\nabla_{t, x} \psi=q=q^{\prime}$ and we get $\mu^{\prime}=\mu$ in step C (14).

Finally, let $(\psi, q, \mu)$ be a fixed point of the algorithm. Let us show that it is a saddle point of $\mathbf{L}$. Step C gives immediately $\nabla_{t, x} \psi=q$, and consequently step B gives $\langle\mu, p-q\rangle \leq 0$ for all $p \in \mathcal{P}$. From step A, we have $G(h)+\left\langle\mu, \nabla_{t, x} h\right\rangle=0$ for all $h \in H^{1}(Q)$. Since the three properties (I) are verified, $(\psi, q, \mu)$ is a saddle point of $\mathbf{L}$ from Proposition 3.1.

It is now possible, according to Proposition 8.1, to reformulate the problem of convergence of the algorithm (12)-(14) to a saddle point of the Lagrangian $\mathbf{L}$, as a problem of convergence to a fixed point of an operator that concatenates the 3 steps of the Benamou-Brenier algorithm. For this purpose, we rely on fixed points of non-expansive operators. This strategy can indeed be used to show the convergence, weak or strong, of proximal splitting algorithms $[21,10,3,6]$.

We consider the space $H=L^{2}(Q)^{d+1} \times L^{2}(Q)^{d+1}$, provided with the scalar product

$$
\left\langle\left(\mu_{1}, q_{1}\right),\left(\mu_{2}, q_{2}\right)\right\rangle_{H}=\left\langle\mu_{1}, \mu_{2}\right\rangle_{L^{2}}+r^{2}\left\langle q_{1}, q_{2}\right\rangle_{L^{2}},
$$

so that $(H,\langle.,\rangle$.$) is an Hilbert space. Let \mathbf{B}: H \rightarrow H$ be the operator which associate to $(\mu, q)$ the product $\left(\mu^{\prime}, q^{\prime}\right)$ of steps B (13) and C (14) of Benamou-Brenier's algorithm. Here $\psi$ just acts as an auxiliary variable. Indeed, if $\left(\mu^{*}, q^{*}, \psi^{*}\right)$ is a saddle point of the Lagrangian $\mathbf{L}$ defined in (9), then $\left(\mu^{*}, q^{*}\right)=\mathbf{B}\left(\mu^{*}, q^{*}\right)$. Conversely if $\left(\mu^{*}, q^{*}\right)$ is a fixed point of $\mathbf{B}$ then $\left(\mu^{*}, q^{*}, \psi^{*}\right)$ is a saddle point of the Lagrangian, where $\psi^{*}$ is the unique element of $S$ which satisfies $q^{*}=\nabla \psi^{*}$.

We then have the following result, demonstrated in Appendix B [19].

Proposition 8.2. Operator $\mathbf{B}$ is non-expansive on $H$ and quasi-firmly non-expansive on $\mathbf{B}(H)$.

As $\mathbf{B}$ is non-expansive, the Benamou-Brenier algorithm weakly converges in $L^{2}$ to a fixed point of $\mathbf{B}$ (i.e. a saddle point of $\mathbf{L}$ ) whose existence is shown by Theorem 3.1. From the quasifirmly non-expansiveness, we can also define a relaxed version of the algorithm with strong- $L^{2}$ convergence. We refer to Appendix B [19] and the results of $\mathrm{H}$. Bauschke in [2] for more details.

Notice that Proposition 8.2 also justifies the convexity (and closure) of the set of saddle points of $\mathbf{L}$. The set of fixed points of a non-expansive operator is indeed a closed convex set. The operator B immediately gives us this convexity for the components $\mu$ and $q$. The characteristic $(\mathrm{I})_{3}$ of the properties (I) as well as the linearity of the gradient operator $\nabla_{t, x}$ transfer this convexity to the component $\psi$, and therefore to the set of saddle points of $\mathbf{L}$.

\section{Conclusion and perspectives}

The starting point of our work is the study of the Lagrangian augmented algorithm of BenamouBrenier [4]. We show in the section 8 the convergence of this algorithm to a saddle point of the Lagrangian $\mathbf{L}$, which models the dynamic formulation of the optimal transport problem.

The convergence of the algorithm is conditioned by the existence of a saddle point for the Lagrangian L. In sections 4 and 5, we thus tackle the problem of existence of such saddle points. In section 6 , we show the uniqueness of the couple density/ momentum. Our proof is based on the properties of regularity and the dynamic structure of the velocity field, whereas previous methods such as [1] or [27] use the energetic formulation and geodesic displacements in Wasserstein's spaces. Our study has been carried out in the most general conditions, especially in cases where the initial and final densities $\rho_{0}$ and $\rho_{1}$ vanish on some subset of the transport domain, which generally involve non-regular optimal transport plans. As far as we know, this is the first convergence proof of the Benamou-Brenier algorithm for vanishing densities in $L^{2}$. 
We also present in Proposition 6.2 new results on the regularity and the control of a velocity field associated to an optimal transport. In Theorem 7.1, we finally exhibit the minimal properties that a velocity field has to satisfy in order to be associated to an optimal transport in $L^{2}$.

In forthcoming works, we would like to analyze the convergence properties of dynamic optimal transport algorithms: stopping or distance criterias with respect to the solution, theoretical information on the speed of convergence, etc... Another perspective concerns the extension of our existence and uniqueness results in $L^{2}$ to generalized dynamic optimal transport settings, especially non-isotropic domains (see [18]) or Riemannian manifolds.

Acknowledgments: This study has been carried out with financial support from the French State, managed by the French National Research Agency ANR TOMMI (ANR 2011 BS01 014 01) and GOTMI (ANR-16-CE33-0010-01). The project has also received funding from Grenoble INP, through SEI grant TOSCANA.

\section{References}

[1] L. Ambrosio, N. Gigli, and G. Savaré. Gradient flows: in metric spaces and in the space of probability measures. Springer Science \& Business Media, 2008.

[2] H. Bauschke. The approximation of fixed points of compositions of nonexpansive mappings in hilbert space. Journal of Mathematical Analysis and Applications, 202(1):150-159, 1996.

[3] H. Bauschke and P. L. Combettes. Convex analysis and monotone operator theory in Hilbert spaces, volume 408. Springer, 2011.

[4] J.-D. Benamou and Y. Brenier. A computational fluid mechanics solution to the MongeKantorovich mass transfer problem. Numerische Mathematik, 84(3):375-393, 2000.

[5] J.-D. Benamou, B. D. Froese, and A. M. Oberman. Numerical solution of the optimal transportation problem using the monge-ampère equation. Journal of Computational Physics, 260:107-126, 2014.

[6] R. I. Boţ, E. R. Csetnek, and D. Meier. Inducing strong convergence into the asymptotic behaviour of proximal splitting algorithms in hilbert spaces. Optimization Methods and Software, 34(3):489-514, 2019.

[7] L. Caffarelli. Boundary regularity of maps with convex potentials. Communications on pure and applied mathematics, 45(9):1141-1151, 1992.

[8] L. Caffarelli. Boundary regularity of maps with convex potentials-ii. Annals of mathematics, 144(3):453-496, 1996.

[9] L. Chizat, G. Peyré, B. Schmitzer, and F.-X. Vialard. Unbalanced optimal transport: Dynamic and kantorovich formulations. Journal of Functional Analysis, 274(11):3090-3123, 2018.

[10] P. L. Combettes and J.-C. Pesquet. A Douglas-Rachford splitting approach to nonsmooth convex variational signal recovery. IEEE Journal of Selected Topics in Signal Processing, 1(4):564-574, 2007.

[11] D. Cordero-Erausquin. Sur le transport de mesures périodiques. Comptes Rendus de l'Académie des Sciences-Series I-Mathematics, 329(3):199-202, 1999. 
[12] R. J. DiPerna and P.-L. Lions. Ordinary differential equations, transport theory and sobolev spaces. Inventiones mathematicae, 98(3):511-547, 1989.

[13] M. Fortin and R. Glowinski. Augmented Lagrangian Methods: Applications to the Numerical Solution of Boundary-Value Problems. Studies in Mathematics and its Applications. Elsevier Science, 1983.

[14] V. Girault and P.-A. Raviart. Finite Element Methods for Navier-Stokes Equations: Theory and Algorithms. Springer-Verlag, 2011.

[15] M. Goldman and F. Otto. A variational proof of partial regularity for optimal transportation maps. arXiv preprint arXiv:1704.05339, 2017.

[16] K. Guittet. On the time-continuous mass transport problem and its approximation by augmented Lagrangian techniques. SIAM Journal on Numerical Analysis, 41(1):382-399, 2004 .

[17] R. Hug. Mathematical analysis and convergence of an algorithm for dynamic optimal transport: case of non-smooth transport plans. PhD thesis, Université de Grenoble, 2016.

[18] R. Hug, E. Maitre, and N. Papadakis. Multi-physics optimal transportation and image interpolation. ESAIM: Mathematical Modelling and Numerical Analysis, 49(6):1671-1692, 2015 .

[19] R. Hug, E. Maitre, and N. Papadakis. Appendix: On the convergence of augmented lagrangian method for optimal transport between nonnegative densities. hal-01128793, 2017.

[20] L. Kantorovitch. On the translocation of masses. Management Science, 5(1):1-4, 1958.

[21] P. L. Lions and B. Mercier. Splitting algorithms for the sum of two nonlinear operators. Siam J. Numer. Anal., 16:964-979, 1979.

[22] D. Lombardi and E. Maitre. Eulerian models and algorithms for unbalanced optimal transport. ESAIM: Mathematical Modelling and Numerical Analysis, 49(6):1717-1744, 2015.

[23] R. McCann. A convexity principle for interacting gases. Advances in mathematics, 128(1):153-179, 1997.

[24] N. Papadakis, G. Peyré, and E. Oudet. Optimal transport with proximal splitting. SIAM Journal on Imaging Sciences, 7(1):212-238, 2014.

[25] G. Peyré and M. Cuturi. Computational optimal transport. Foundations and Trends® in Machine Learning, 11(5-6):355-607, 2019.

[26] W. Rudin, N. Dhombres, and F. Hoffman. Analyse réelle et complexe. Masson Paris, 1975.

[27] F. Santambrogio. Optimal transport for applied mathematicians. Birkäuser, NY, 55:58-63, 2015 .

[28] C. Villani. Topics in Optimal Transportation. American Mathematical Society, 2003.

[29] C. Zuily. Éléments de distributions et d'équations aux dérivées partielles: cours et problèmes résolus. Dunod, 2002. 